\title{
[Oral Presentation]
}

\section{0-1 \\ Development of an online system to efficiently handle animal experiment plans}

\author{
Shuuji Tsuchiyama, Naomi Nakagata
}

Division of Reproductive Engineering, Center for Animal Resources and Development (CARD), Kumamoto University, Kumamoto, Japan

Institutional Animal Care and Use Committee (IACUC) reviews animal experiment plans based on animal experiment rules and regulations, and approves plans which comply with the rules. Interaction between researchers and IACUC members should be smooth to improve the compliance of animal experiment plans. At our institute, we normally conduct a paper-based review of animal experiment plans which can be a bottleneck. In response, we created two online systems. The first was developed inhouse using Java, PostgreSQL and Tomcat. Users can create, submit and revise their plans, and IACUC can review, amend and approve them. Furthermore, after an experiment has been completed, reports can be created therein. The second is a commercially available internal wiki system supported by Kumamoto University called Confluence. All staff and students can use the system to create web pages, upload files and leave comments. There is also a collaborative space reserved for IACUC to share and leave comments concerning animal experiment plans. The first system covers almost all information concerning animal experiments and contains many functions, but is rather complicated. Meanwhile, the second system allows the sharing of animal experiment plans and enables comments. To launch a system to support animal experiment, we will use the Confluence system to ascertain which functions are essential, while at the same time making improvements to our own in-house system.

\section{0-2 Introduction of BSL3 laboratory at ICLAS monitoring center, CIEA}

\author{
Tomoko Ishida ${ }^{1}$, Mai Tanaka ${ }^{1}$, Kumiko Yoshimatsu ${ }^{2}$, Akira Takakura ${ }^{1}$, \\ Jiro Arikawa $^{2}$, Nobuhito Hayashimoto ${ }^{1}$ \\ ${ }^{1}$ ICLAS Monitoring Center, Central Institute for Experimental Animals (CIEA) ${ }^{2}$ Graduate School of \\ Medicine Department of Microbiology, HOKKAIDO UNIBERSITY
}

Hantavirus is causative agent of zoonotic infection. Therefore it is tested as a routine test of microbiological monitoring for laboratory rodents. Serology is common methods to test Hantavirus. Antigen derived from whole virion is an essential material for serology. However Hantavirus is classified in Category IV Infectious
Diseases pathogens and their handling is strictly regulated by a low. Recently, we established a Bio Safety Level (BSL) 3 laboratory to produce antigen of Hantavirus. In this presentation, we introduce of this BSL3 laboratory and preparation of antigen of Hantavirus. 


\title{
Examination of material compatibilities with ionized and vaporized hydrogen peroxide disinfection
}

\author{
Tohru Kimura ${ }^{1}$, Hiroyuki Yahata ${ }^{2}$, Yoshimichi Uchiyama ${ }^{2}$, Hironobu Ichihara ${ }^{2}$ \\ ${ }^{1}$ Joint Faculty of Veterinary Medicine, Yamaguchi University, Yamaguchi, Japan ${ }^{2}$ Santasalo \& Steri-pro \\ Solution Corporation, Kobe, Japan
}

Excellent disinfection method with hydrogen peroxide has deleterious effects on some materials after exposure to hydrogen peroxide. The purpose of this study was to examine material compatibilities with ionized and vaporized hydrogen peroxide (IHP and VHP). Various materials were exposed to IHP or VHP disinfection in the test chamber. IHP disinfection: Some damage was found in copper, brass, chromium plate and galvanized iron immediately following exposure to IHP disinfection. Repeated IHP disinfection caused marked damage in stainless steel and urethane-, silicone- or epoxy-coating materials. Condensation of IHP disinfection posed severe damage for the surface of materials. VHP disinfection: In dry conditionings, some resins began to develop hardening or softening. We found discoloration in the stainless steel and changes in its coating materials. In process of VHP disinfection, condensation of VHP caused material damage such as corrosion of zincplated steel and air-bubbling over the colorsteel sheet. The increasing concentrations of IHP with condensation caused severe changes in the materials during prolonged exposure. The results of VHP disinfection provided evidence that the material damage was severer in dry conditions than in condensation conditions. Our results demonstrated that it was highly important to disinfect equipment avoiding condensation of hydrogen peroxide.

\section{0-4}

The antibiotic effect of paromomycin sulfate to enteric protozoa in mice

\author{
Shinichiro Nakamura, Ichiro Terakado \\ Research Center for Animal Life Science, Shiga University of Medical Science
}

Several species of enteric protozoa in mice without pathogenicity are obstacles for the transportation and hygiene management. Paromomycin sulfate (PS) could be used to treat Entamoeba histolytica in humans and Balantidium coli in nonhuman primates (NHP). We confirmed whether PS revealed antibiotic effect to enteric protozoa in mice.

Male 4-weeks(W)-old C57BL/6J mice were bred for 6 months with decoys of microbiological monitoring in the anteroom, where was detected Chilomastix bettencourti and Octomitus intestinalis, and then moved to IVC, with PS in bottled water ad libitum. The dose and duration in each group $(\mathrm{n}=3)$ were control $3 \mathrm{~W}, 0.5 \mathrm{mg} /$ $\mathrm{ml} 2 \mathrm{~W}, 5 \mathrm{mg} / \mathrm{ml} \mathrm{2W}, 5 \mathrm{mg} / \mathrm{ml} 3 \mathrm{~W}$, and $10 \mathrm{mg} /$ $\mathrm{ml} 3 \mathrm{~W}$. They stayed there for $2 \mathrm{~W}$ after final administration. After euthanization, cecum contents were used for microscopic observation. Positive mice were found $3 / 3$ in control, $3 / 3$ in $0.5 \mathrm{mg} / \mathrm{ml} 2 \mathrm{~W}, 1 / 3$ in $5 \mathrm{mg} / \mathrm{ml} 2 \mathrm{~W}, 0 / 3$ in $5 \mathrm{mg} /$ $\mathrm{ml} 3 \mathrm{~W}$, and $0 / 3$ in $10 \mathrm{mg} / \mathrm{ml} 3 \mathrm{~W}$. No fecal and pathological changes were found during the experiments.

The complete antibiotic effect could be obtained by total $525 \mathrm{mg} /$ head administration. While, the precise effect to $B$. coli in NHP were found with total $250 \mathrm{mg} /$ head administration. The antibiotic sensitivities might be different among species of protozoa. This concentration to mice seems to be higher than that to monkeys. However, all mice did not show any side effects, because PS is not absorbed from intestines, and attacks to RNA in protozoa. This is thought to be a valuable antiparasite method. 


\title{
Evaluation of Environmental Enrichment in a Laboratory Canine Breeding Facility
}

\author{
$\bigcirc$ Hiroaki Abe ${ }^{1,2}$, Nicole Navratil ${ }^{2}$ \\ ${ }^{1}$ Marshall BioResources Japan Inc., Tsukuba, Japan ${ }^{2}$ Marshall BioResources, North Rose, United State
}

Environmental enrichment and socialization of laboratory beagles must be carefully considered, especially at a breeding facility with several hundred or several thousand animals, as this environment can pose both spatial and temporal restrictions. Innovation is required to provide variability and complexity within the environment. Items such as toys, must be easily sanitized, durable and safe for a large number of animals. However, this must be balanced with the fact that environmental enrichment also needs to provide constructive outlets for biologically derived behavioral tendencies, such as foraging, rest, exercise and play. A bare environment and lack of stimulation can lead to stress and problematic behaviors such as destructive chewing, aggressive or fearful behaviors, repetitive or obsessive behaviors such as circling or excessive licking, or excessive barking. Prolonged stress is an animal welfare concern and can lead to physiological changes that can reduce the reliability of study results. The environmental enrichment and socialization program at Marshall is multifaceted and involves the use of toys, various resting surfaces, social housing, and complexity in the cage design to provide choice and simulation, as well as space for exercise and play. This poster describes ongoing evaluation of our enrichment program, how the dogs utilize their environment, the observed behavioral patterns and social activity, and what preferences the dogs seem to have.

\section{0-6 \\ Analysis system of sperm fertility by flow cytometer using microfluidics chip}

\author{
$\bigcirc$ Satohiro Nakao ${ }^{1}$, Toru Takeo ${ }^{1}$, Hitomi Watanabe ${ }^{2}$, Gen Kondo ${ }^{2}$, Naomi Nakagata ${ }^{1}$ \\ ${ }^{1}$ Division of Reproductive Engineering, Center for Animal Resources and Development, Kumamoto University, Kumamoto, Japan \\ ${ }^{2}$ Laboratory of Integrative Biological Science, Institute for Frontier Life and Medical Sciences, Kyoto University, Kyoto, Japan
}

Sperm selection is an important event to complete fertilization in female reproductive tract. In in vitro fertilization (IVF), synthesized fertilization medium activates sperm to induce capacitation. However, many sperm should be inseminated to fertilize egg in the IVF $(>200$ sperm/L). Investigating the mechanism of sperm selection in female reproductive tract may be useful to improve the efficiency of fertilization using the small number of sperm. In this study, we selected capacitated sperm labeled with Lectin-PNA-FITC by flow cytometer using microfluidics chip and examined the motility and fertility of the capacitated and un-capacitated sperm after the sorting. The sorted-sperm maintained motility between capacitated and un-capacitated group. The fertilization rate of capacitated sperm was higher than that of uncapacitated sperm. Produced embryos derived from the capacitated sperm normally developed to live pups. In summary, we established novel analysis system of sperm fertilizing ability by flow cytometer using microfluidics chip. 


\title{
Development of a controllable Mouse Model for Pressure- overload Heart Failure
}

\author{
Masaaki Ii, YOSHINORI KOISHI, CHINATSU SHIRAOKA, SHINTARO NEMOTO \\ Division of Research Animal Laboratory and Translational Medicine, Osaka Medical College
}

Transverse aortic constriction (TAC) surgery is commonly used for making mouse pressure overload heart failure (HF), however, advanced technique is required and it is known to be difficult to induce stable HF in each mouse. We performed TAC surgery in mice by tying transverse aorta with a 6-0 silk suture under monitoring systolic blood pressure (SBP) of right carotid artery using a 0.9Fr optical pressure catheter. Echocardiography and statistical analysis showed inverse correlation $(R=0.660$, $\mathrm{n}=23$ ) between factional shortening (FS) and SBP ratio, and positive correlation $(\mathrm{R}=0.567$, $n=23$ ) between $L V d D$ and SBP ratio. The SBP ratios of 1.15-1.25 and 1.45-1.55 resulted in left ventricular hypertrophy (LVH) with normal contraction and HF with left ventricular dilatation, respectively. We have developed a stable/controllable mouse model for LVH and HF by adjusting SBP ratio before and after TAC surgery.

\section{0-8 Open Wet-dressing Therapy (OWT) for Skin Wounds in Two Cynomolgus Monkeys}

\author{
Toshiki Iwasaka, Hroyuki Izumi
}

Shin Nippon Biomedical Laboratories, Ltd., Kagoshima, Japan

(Aim)OWT is a moist wound healing therapy that promotes exudation in semi-occlusive environments. It has been shown to be simple, economical and effective in treating wounds in humans and animals using Vaseline applied to a pet sheet in a plastic bag (or Pad), but there is little research in experimental animals. One challenge with primates is discouraging dressing removal. (Methods)Case 1:Wounds in the right arm were observed in a 3-year-old male. Surgery was conducted 7 days later under anesthesia. The upper arm wound was stitched, while the swollen forearm wound was left open. Wounds were coated with pads. A linen arm cover was fixed to the shoulder of the jacket using zip ties. Case 2:Wounds from self-biting were observed in both legs in a 4-year-old male. Surgery was conducted 2 days later under anesthesia. The right leg wounds were stitched and the left calf wounds were left open. Affected areas were coated with pads. The animal was dressed in pediatric overalls and a jacket to prevent dressing removal. (Results)Pads were replaced weekly and removed after 3 weeks (Case 1) or 2 weeks (Case 2). In both cases, the wounds healed completely after 5 weeks. (Discussion) Cynomolgus monkeys are dexterous and can exacerbate wounds by touching them. Wound deterioration due to self-biting is occasionally observed. We found OWT facilitated wound healing in two cynomolgus monkeys. Jackets with arm covers or pediatric overalls prevented pad removal, suggesting they were effective in preventing self-injury. 


\title{
0-9 Anesthetic management for neonatal pig.
}

\author{
$\bigcirc$ Takuya Ito ${ }^{1}$, Juntaro Shiba ${ }^{2}$, Takashi Igarashi ${ }^{2}$, Kamori Igarashi ${ }^{1}$, Noriko Handa ${ }^{1}$, Shuji Hishikawa ${ }^{1}$, Hiroaki Shibata ${ }^{1}$, Hideki Uosaki ${ }^{3}$, \\ Hiromasa Hara ${ }^{3}$, Tooru Wakuil, Kazushi Miyazawa', Mizuki Kurita', Masanori Ito', Yutaka Hanazono ${ }^{1,3}$, Satoshi Kunita ${ }^{1}$ \\ ${ }^{1}$ The Center of Development of Advanced Medical Technology, Jichi Medical University, Shimothuke, Japan ${ }^{2}$ Anesthesiology \& Critical Care Medicine, Jichi \\ Medical University, Shimothuke, Japan ${ }^{3}$ Division of Regenerative Medicine, Center for Molecular Medicine, Jichi Medical University, Shimothuke, Japan
}

Establishment of anesthetic management for neonatal pigs is necessary for development of treatment for disease. However, the development of therapy involving intervention for neonates has been at a standstill because an anesthetic procedure for neonatal pigs has not been established. Here, we report successful anesthetic management in neonatal pigs. A 14-day and a 1-month old pigs were used. Anesthesia for those pigs was performed as paying attention to prevent hypotension, hypoglycemia and hypothermia. Five-percent glucose lactated Ringer's solution was infused from vein for maintenance. A bolus of the solution was given to maintain blood pressure as volume of bleeding and blood sampling were measured. After the adjustment of infusion volume and sevoflurane concentration while monitoring the blood pressures, their spontaneous respirations were confirmed 20-40 minutes after closing wounds as arousals from anesthesia were achieved. Surgical interventions for neonatal pigs are implemented. The results and design recommendations will provide the direction to develop treatment various diseases for neonatal pigs.

\section{0-10 Establishment of method for analyzing the first wave of spermatogenesis in mice.}

\author{
$\bigcirc$ Yuki Osawa ${ }^{1,2}$, Abdelaziz Elzeftawy ${ }^{1}$, Yoko Daitoku ${ }^{1}$, Yoshikazu Hasegawa ${ }^{1}$, \\ Ken-ichi Yagami ${ }^{1}$, Seiya Mizuno ${ }^{1}$, Fumihiro Sugiyama ${ }^{1}$ \\ ${ }^{1}$ Laboratory animal science, University of Tsukuba, Ibaraki, Japan ${ }^{2}$ Department of Biology, University \\ of Tsukuba, Ibaraki, Japan
}

In matured male spermatogenesis, there are 12 stages in which each stage has a different combination of germ cells in seminiferous tubule. Sperm is developed continuously and synchronously. It is possible to find male germ cells at a specific differentiation stage by observing the testes of a specific age in the first spermatogenesis called first wave. More than 2,300 genes are believed to be expressed predominantly in male germ cells. To investigate these genes function using genes modified mouse strains, it is important to establish the methods of the first wave analyses. In this time, we made sure the suitable fixing condition for juvenile mice testis (day post portum (dpp)3, dpp8, dppl4, dpp20). Each testis was fixed by Bouin's solution, 4\% Paraformaldehyde, or 10\% Formalin, and embedded in OCT (frozen) or paraffin. Then, we performed HE staining and immunofluorescence of several marker protein and the results suggested that Bouin fixation for HE staining and Formalin fixation for immunofluorescence are suitable. 

marmoset

\author{
$\bigcirc$ Keiko Kishimoto ${ }^{1}$, Akiko Shimada ${ }^{2}$, Tsukasa Takahashi ${ }^{1}$, Haruka Shinohara ${ }^{1}$, \\ Yasuhiro Takashima ${ }_{2}$, Erika Sasaki ${ }^{1}$ \\ ${ }^{1}$ CIEA, Kanagawa, Japan ${ }^{2}$ CiRA, Kyoto, Japan
}

Embryonic stem cells (ESCs) are the useful tool for the study of in vitro embryonic development. Although many embryonic developmental studies performed using mouse ESCs, there are differences between mouse ESCs and primate ESCs. Currently, pluripotent stem cells including ESCs are classified into two states, naive and primed. Mouse ESCs which are able to contribute to chimeric animals are called as naive state, on the other hand, primate that don't have competency for contribution to chimeric animals are thought to be similar to post implanted epiblast and it is called primed state. To understand the differences, nonhuman primate ESCs would be good models. Especially, the common marmoset monkey (Callithrix jacchus) is a useful model because of their short generation period and delivers twins or triplets for each pregnancy. Although several ESC lines were derived from the common marmoset embryos, recently new ESC lines have not been established. In this study, we established and characterized of novel common marmoset ESC lines. First, we cultured the inner cell mass collected from embryos by immunosurgery on the mouse embryonic fibroblast or on iMatrix (laminin fragments). Then, we characterized these ESC lines by gene expression analyses and karyotype analysis. Moreover, we succeeded in establishing ESC lines from not only natural mating but also in vitro fertilized embryos. The novel marmoset ESC lines will promote basic primate embryo and ESCs studies.

\title{
0-12 Naive-like conversion of Cynomolgus Monkey ES/iPS cells
}

\author{
Arata Honda ${ }^{1,2}$, Yoshihiro Kawano ${ }^{3}$, Haruna Izu $^{3}$, Narantosog Choijookhuu ${ }^{3}$, Tomonori Nakamura ${ }^{1}$, \\ Yasuhiro Takashima ${ }^{4}$, Michiko Hirose ${ }^{2}$, Tadashi Sankai ${ }^{5}$, Yoshitaka Hishikawa ${ }^{3}$, Atsuo Ogura ${ }^{2}$, Mitinori Saitou ${ }^{1,4}$ \\ ${ }^{1}$ Kyoto University Graduate School of Medicine, Kyoto, Japan ${ }^{2}$ RIKEN BioResource Center, Ibaraki, Japan. ${ }^{3}$ University of Miyazaki, Miyazaki, Japan ${ }^{4}$ Center for \\ iPS Cell Research and Application, Kyoto University, Kyoto, Japan ${ }^{5}$ Tsukuba Primate Research Center, National Institute of Biomedical Innovation,
}

When the derivation of so-called true naive state induced pluripotent stem cells (iPSCs) can be achieved, this will be of great benefit to regenerative medicine by the generation of differentiated tissues and/or cells in vitro as well as whole organs in live animals. In this report, we have demonstrated and evaluated cynomolgus monkey $(\mathrm{Cm})$ (Macaca fascicularis) embryonic stem cells (Cm ESCs) and Cm iPSCs before and after naive-like conversion. This report offers clues for generating and evaluating true naive PSCs in a primate model. After determination of the culture conditions needed to convert prime state Cm ESCs to naive-like state, Cm iPSCs were established and then converted to a naive-like state as Cm ESCs. These naive-like converted Cm PSCs showed several characteristics of a naive-like state such as colony morphology, pluripotency, and enhanced capacity for in vitro differentiation. However, experiments involving transfer to host mouse embryos and evaluating the transcriptomal status of naive-like converted PSCs revealed that they did not convert to a true naive state completely but only partially. 


\title{
O-13 Pluripotent stem cells in disease modelling of GJB2 related hearing loss
}

\author{
$\bigcirc$ Kazusaku Kamiya, Ichiro Fukunaga, Ayumi Fujimoto, Katsuhisa Ikeda \\ Juntendo University, Tokyo, Japan
}

Mutation of the Gap Junction Beta 2 gene (GJB2) is the most frequent cause of hereditary deafness worldwide and accounts for up to $50 \%$ of nonsyndromic sensorineural hearing loss cases in some populations. GJB2 encodes connexin (CX) 26, a component in cochlear gap junction. We have demonstrated that the drastic disruption of gap junction plaque (GJP) macromolecular complex composed of CX26 and CX30 are critical pathogenesis starting before hearing onset (Kamiya, J Clin Invest, 124(4):1598-1607, 2014). Therefore, cochlear CX26-gap junction plaque (GJP)-forming cells such as cochlear supporting cells are thought to be the most important therapeutic target for the treatment of hereditary deafness. In this study, we developed a novel strategy to differentiate induced pluripotent stem cells into functional CX26-GJPforming cells that exhibit spontaneous ATP- and hemichannel-mediated Ca2+ transients typical of the developing cochlea. Furthermore, these cells from CX26-deficient mice recapitulated the drastic disruption of GJPs, the primary pathology of GJB2-related hearing loss (Fukunaga, Stem Cell Reports, 7(6), 1023-1036, 2016). Human iPS cells were also developed form the patients with common GJB2 mutations in east Asian population. These in vitro models should be useful for establishing inner-ear cell therapies and drug screening that target GJB2-related hearing loss.

\section{0-14 Development of hemato-lymphoid chimeric mice by using intraplacental transplantation}

\author{
$\bigcirc$ Hyojung Jeon, Keigo Asano, Michito Hamada, Satoru Takahashi \\ Dept. of Anatomy and Embryology, Univ. of Tsukuba, Japan
}

Background: In experimental hematopoietic stem cell (HSC) transplantation, the method of HSC reduction for embryo and perfect reconstruction of host hematopoiesis by donor cells has not been reported. The purpose of this study is to generate new hemato-lymphoid chimeric mice by using intraplacental transplantation.

Method: To establish the method of HSC injection in mouse embryo, we transplanted donor HSC into the embryo stage of transgenic mice that have no HSC. We used mouse, rat fetal liver cell and human umbilical cord blood cells as donor cells. The fetal liver of recipient embryos was obtained in E18.5 and examined the chimerism by FACS.

Result: We observed more than $80 \%$ of donor cells chimerism in E18.5 fetal liver of recipient embryo. Moreover, donor cells in recipient embryo were differentiated into myeloid, B and $\mathrm{T}$ cell lymphoid cells. In rat derived cell transplantation, higher chimerism of donor cells were observed in recipient mice embryo. FACS and colony assay showed donor fetal liver hematopoietic cells contributed to both white blood cells and red blood cells. When we used human cells as a donor, we could not be detected donor derived cell from recipient embryo in E18.5. But we confirmed few donorderived cells in 4 weeks old mice peripheral blood. These results suggest that the mouse lacking fetal liver HSC is a powerful tool for hematopoiesis reconstruction during embryonic day and potentially be applied to basic research of HSC or xenograft models. 


\title{
0-15 Influence of SLA class II haplotypes on reproductive traits in a miniature pig line, Microminipig
}

\author{
$\bigcirc$ Asako Ando ${ }^{1}$, Noriaki Imaeda ${ }^{2}$, Tatsuya Matsubara ${ }^{2}$, Masaki Takasu², Asuka Miyamoto ${ }^{1}$, Shino Ohshima ${ }^{1}$, \\ Takashi Nishimura $^{3}$, Toshiaki Nishimura ${ }^{3}$, Naohito Nishii ${ }^{2}$, Yoshie Kametani ${ }^{1}$, Hitoshi Kitagawa ${ }^{2}$ \\ ${ }^{1}$ Dept. of Molec. Life Sci., Div. of Basic Med. Sci. and Molec. Med., Tokai Univ. Sch. of Med., Isehara, Japan ${ }^{2}$ Dept. of Vet. Med., \\ Fac. of Appl. Biol. Sci., Gifu Univ., Gifu, Japan ${ }^{3}$ Fuji Micra Inc., Fujinomiya, Japan
}

Using swine leukocyte antigen (SLA)-defined pigs, influence of SLA-encoded genes on reproductive traits such as litter sizes has been reported. We have previously assigned eight SLA class II haplotypes in a miniature pig line named as Microminipig (MMP). In this study, to clarify the involvement of SLA class II genes in reproductive traits in MMPs, we compared four reproductive performance; female fertility index and abnormal delivery, gestation periods and litter sizes, among SLA class II haplotypes (Hps) of 132 dams or 58 sires in 1146 deliveries using multiple comparison tests. The fertility index of dams with Hp-0.13 was significantly lower than that of dams with Hp-0.16, 0.17, 0.18, or 0.37. Dams with Hp-0.23 had significantly smaller litter sizes than those with $\mathrm{Hp}-0.17,0.18$, or 0.37. Furthermore, litter sizes except stillbirth and miscarriage of dams with Hp-0.23 were also significantly smaller than those of dams with Hp$0.17,0.18$, or 0.37 . The small litter size of dams with Hp-0.23 might reflect the small body sizes of MMPs with Hp-0.23. These results suggest that SLA class II haplotypes might be useful makers as selective breeding in MMPs.

\section{0-16 Identification of a new quantitative trait gene for resistance to obesity in wild mice}

\author{
$\bigcirc$ Akira Ishikawa, Keita Makino \\ Graduate School of Bioagricultural Sciences, Nagoya University, Nagoya, Japan
}

Our previous QTL analysis using C57BL/6 (B6) inbred mice and wild Mus musculus castaneus mice derived from the Philippines revealed multiple QTLs for growth related traits in a 44$\mathrm{Mb}$ genomic interval of chromosome 2. At one of these QTLs, a wild-derived allele uniquely showed resistance to obesity. Subsequent congenic analysis, RNA-seq analysis, causal analysis and others revealed a single candidate gene for the obesity-resistant QTL. In this study, we identified a causal gene, called a quantitative trait gene (QTG), for the obesity-resistant QTL by two mating experiments using knockout (KO) mice for the candidate gene. One experiment is quantitative complementation tests using fourway crosses among the $\mathrm{KO}$ strain, a congenic strain carrying the wild-derived QTL allele on the
B6 background and their background B6 strains. The other experiment is phenotypic analysis and real-time PCR analysis using $\mathrm{F}_{2}$ intercross mice between KO and B6 strains. Quantitative complementation tests indicated a statistical, marginal interaction effect between genotypes of the KO locus and the QTL. Phenotypic analysis revealed that white fat pad weight of mice homozygous for the B6-derived allele was significantly lighter than that of mice hemizygous for B6 and KO alleles. Real-time PCR analysis indicated a significant negative correlation between expression of the candidate gene and fat pad weight. The results strongly suggested that the candidate gene is a new QTG for resistance to obesity in wild mice. 

mammalian functional genomics

\author{
Toyoyuki Takada ${ }^{1}$, Kentaro Fukuta ${ }^{2}$, Hideki Noguchi ${ }^{2}$, Atsushi Toyoda ${ }^{2,3}$, \\ Shoko Kawamoto ${ }^{4}$, Toshihiko Shiroishi ${ }^{1}$ \\ ${ }^{1}$ Mammalian Genetics Laboratory, National Institute of Genetics ${ }^{2}$ Advanced Genomics Center, National Institute of Genetics \\ ${ }^{3}$ Comparative Genomics Laboratory, National Institute of Genetics ${ }^{4}$ Genetic Informatics Laboratory, National Institute of Genetics
}

National Institute of Genetics (NIG) has established a series of experimental mouse strains named "Mishima Battery". It consists of ten inbred strains originated from four mouse subspecies including the Japanese subspecies, which are widely distributed in the world. Our recent genome-resequencing project of " Mishima Battery" revealed over forty millions of SNPs due to large inter-subspecific genetic distance. We expected that information of the genome polymorphisms could be applied not only to study the natural history of this species but also to perform gene discovery of QTLs affecting complex traits based on the inter-subspecific genome diversity. Recently, we have released an upgraded version of the NIG Mouse Genome database named "NIG_MoG2". NIG_MoG2 primarily comprises the whole genome sequence data of the "Mishima Battery", providing visualized genome polymorphism information with single-nucleotide polymorphisms and short insertions/deletions in the genomes of the " Mishima Battery". In this talk, we will present results of our recent project that aims to explore physiological phenotypes associated genes by functional genomics with the "Mishima Battery".

\title{
0-18 Forward genetics approach reveals the tumor-suppressive effect of parathyroid hormone
}

\author{
$\bigcirc$ Kazuhiro Okumura ${ }^{1}$, Megumi Saito ${ }^{1}$, Eriko Isogai ${ }^{1}$, Ikuo Miura ${ }^{2}$, Shigeharu Wakana ${ }^{2}$, \\ Midori Yamaguchi ${ }^{3}$, Hiroshi Shitara ${ }^{3}$, Choji Taya ${ }^{3}$, Ryo Kominami ${ }^{4}$, Yuichi Wakabayashi ${ }^{1}$

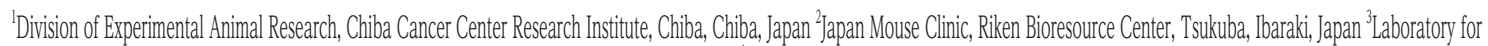

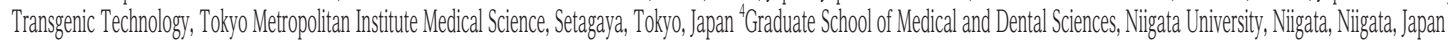

Using a forward genetics approach to map loci in a mouse skin cancer model, we previously identified a genetic locus, Skin tumor modifier of MSM 1 (Stmm) on chromosome 7, conferring strong tumor resistance. Here, we report that serum intact-PTH (iPTH) and a genetic polymorphism in Pth are important for skin tumor resistance. We identified higher iPTH levels in sera from cancer-resistant MSM/Ms mice compared with susceptible FVB/NJ mice. Therefore, we performed skin carcinogenesis experiments with MSM-BAC transgenic mice
(Pth $^{\text {MSM }}$ - Tg) and Pth knockout heterozygous mice $\left(P t h^{+/}\right)$. As a result, the higher amounts of iPTH in sera conferred stronger resistance to skin tumors. Furthermore, we found that the coding SNP (rs51104087, Val28Met) localizes in the mouse Pro-PTH encoding region, which is linked to processing efficacy and increased PTH secretion. Finally, we report that PTH increases intracellular calcium in keratinocytes and promotes their terminal differentiation. Taken together, our data suggest that Pth is one of the genes responsible for Stmml. 


\title{
0-19 Genetic analysis of tameness using selectively bred mice made from wild-derived heterogeneous stock
}

\author{
Yuki Matsumoto ${ }^{1,2}$, Hiromichi Nagayama ${ }^{1,2}$, Akira Tanave ${ }^{1}$, Tatsuhiko Goto ${ }^{3}$, \\ Tsuyoshi Koide ${ }^{1,2}$ \\ ${ }^{1}$ Mouse Genomics Resource Laboratory, National Institute of Genetics, Mishima, Japan ${ }^{2}$ Department of Genetics, SOKENDAI, Mishima, \\ Japan ${ }^{3}$ Research Center for Global Agromedicine, Obihiro University of Agriculture and Veterinary Medicine, Hokkaido, Japan
}

Tameness is a major behavioral factor for domestication, and is a behavioral characteristic with two potential components: reluctance to avoid humans (passive tameness) and motivation to approach humans (active tameness). In our study, we tried to elucidate genetic and neural mechanism underlying active tameness by conducting selective breeding using genetically diverse wild-derived heterogeneous stock (WHS) made from eight wild inbred strains. As a result of selective breeding of the wild-derived heterogeneous stock, the level of contacting increased through the generations. Applying selection mapping to the selected population using a simulation based on a non-selection model and inferred haplotype data derived from single-nucleotide polymorphisms, we found a genomic signature of selection on chromosome 11. Two closely located loci are syntenic to the dog genomic regions which are known to be regions selected during dog domestication, suggesting that it is associated with active tameness in both mouse and dog. Our current attempts to analyze RNA transcriptome will provide information for molecular basis of active tameness in mice.

\section{0-20 Identification of candidate genes for generalized tonic-clonic seizures in NER}

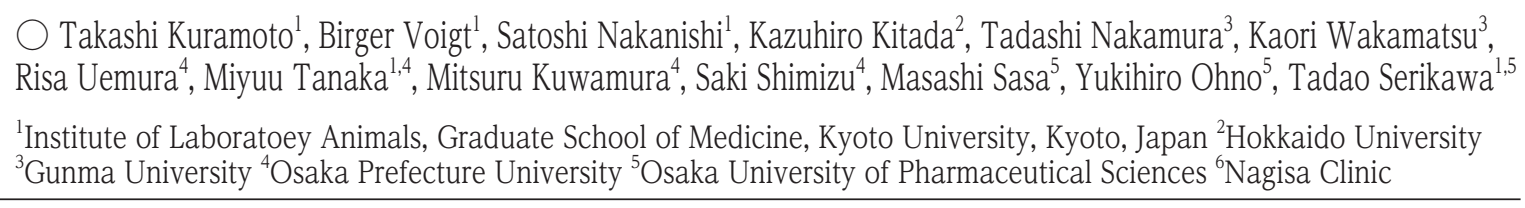

The Noda epileptic rat (NER) exhibits generalized tonic-clonic seizures (GTCS). A genetic linkage analysis identified two GTCS-associated loci, Nerl on Chr 1 and Ner3 on Chr 5. The wildtype Ner1 and Ner3 alleles suppressed GTCS when combined in double-locus congenic lines, but not when present in single-locus congenic lines. Global expression analysis revealed that cholecystokinin B receptor (Cckbr) and suppressor of tumorigenicity 5 (St5), which map within Nerl, and PHD finger protein 24 (Phf24), which maps within Ner3, were significantly downregulated in NER. De novo BAC sequencing detected an insertion of an endogenous retrovirus sequence in intron 2 of the Phf24 gene in the NER genome, and PHF24 protein was almost absent in the NER brain. Phf24 encodes a G $\alpha$ i-interacting protein involved in GABAB receptor signaling pathway. Based on these findings, we conclude that Cckbr, St5, and Phf24 are strong candidate genes for GTCS in NER. 


\title{
0-21 Exploring permissive loci for targeted transgenesis in the mouse
}

\author{
$\bigcirc$ Hirotake Ichise, Taeko Ichise, Nobuaki Yoshida \\ Laboratory of Developmental Genetics, Center for Experimental Medicine and Systems Biology, \\ Institute of Medical Science, University of Tokyo, Tokyo, Japan
}

The introduction of a transgene into a genomic safe harbor through homologous recombination or sequence-specific enzymatic modification is a key technique for producing transgenic mice. The Rosa26 gene has been widely used to produce transgenic mice because the gene is transcriptionally active in various cell types and, at many developmental stages, is permissive for constitutive expression of integrated transgenes. However, permissive loci other than Rosa26 are needed to generate mice that harbor multiple transgenes for complex studies.
In order to explore for good alternatives to Rosa26, we identified transgene integration sites in transgenic mouse lines showing widespread reporter expression, and demonstrated that $C d 6$ is valuable as an alternative site for targeted transgenesis (Ichise, 2014). We also found that Rprdlb permits widespread expression of an integrated transgene (Ichise, 2016). In this presentation, we will describe our published and unpublished data on the candidate loci for targeted transgenesis.

\section{0-22 Whole exome sequencing identifies a novel Criml mutant mouse line}

\author{
Tatsuya Furuichi ${ }^{1}$, Manami Tsukamoto ${ }^{1}$, Yuriko Sato ${ }^{1}$, Masaki Saito ${ }^{1}$, \\ Ryutaro Fukumura ${ }^{2}$, Yoichi Gondo ${ }^{2}$ \\ ${ }^{1}$ Co-Department of Veterinary Medicine, Iwate University, Morioka, Japan ${ }^{2}$ RIKEN Bioresource Center, \\ Tsukuba, Japan
}

We identified a mouse mutant that exhibits microphthalmia and hindlimb syndactyly from a ENU mouse mutagenesis screening. By crossing experiment, these phenotypes were shown to be inherited as an autosomal recessive trait. We named this mutant allele as imla (Iwate microphthalmia with limb anomalies) and tried to identify it by whole exome sequencing. By using SureSelectXT Mouse All Exon Kit, we concentrated exon and its boundary regions from imla homozygote's genome and sequenced them using a next-generation sequencer, Ion ProtonTM system. We identified 71 mutations that might change the coded protein functions. By searching for the phonotypes of 71 mouse mutants, we knew that the phonotypes of Crim 1 mutants were very similar to those of imla homozygotes, suggesting that Criml was the causative gene. The identified Criml mutation, c.418T $>$ A, was a missense mutation that causes a Cys-toSer substitution at position 140 (p.Cysl40Ser). Linkage analysis showed that the two markers located near Crim 1 region completely linked to imla phenotypes. These results strongly suggest that imla is the Criml mutation, p.Cysl40Ser. Because imla homozygous mice exhibit novel phenotypes as Criml mutants, this novel mutant is useful to examine novel CRIMl functions. 

retroelement in agouti gene

\author{
Akira Tanave ${ }^{1}$, Tsuyoshi Koide ${ }^{1,2}$ \\ ${ }^{1}$ Mouse Genomics Resource Laboratory, National Institute of Genetics, Mishima, Shizuoka, Japan \\ ${ }^{2}$ Department of Genetics, SOKENDAI, Mishima, Shizuoka, Japan
}

$<$ Aim $>$ Coat color mutations are common and remain useful tool today for genetics in laboratory animals. Although the nonagouti (a) mutation on agouti locus causes black hairs, the details of the genetic mutation and the history remain obscure. Here, we identified the actual reason of the phenotypic change and revealed the genetic history based on the formation of the a mutation. $<$ Methods $>$ Using the B6 reference genome sequence and the NGS-data of several laboratory strains, we performed molecular genetic and molecular phylogenetic analyses of the agouti gene. We developed a phenotypic revertant of the black hair via CRISPR/Cas9 system. $<$ Results and Discussion $>$ We identified a complex structure of the retroelement in the a allele, in which a part of the sequence was assumed to be an actual cause of the black hair. A deletion of the sequence was enough to revert the phenotypic mutation. Furthermore, phylogenetic analyses of the agouti genes revealed that the $a$ allele has a similar haplotype with that of mice of certain Mus musculus subspecies. These findings indicate that the a allele had been developed by a structural change of the retroelement in the agouti gene. We conclude that the a alleles of common laboratory mice are derived from certain black mice.

\title{
0-24 Increased expression of Lrrc30 suppresses the onset of age- related hearing loss in mice
}

\author{
$\bigcirc$ Yoshiaki Kikkawa $^{1}$, Yuki Miyasaka ${ }^{1,2}$, Sumpei P Yasuda ${ }^{1}$, Sari Suzuki ${ }^{1,3}$, Hiroshi Shitara ${ }^{1}$ \\ ${ }^{1}$ Tokyo Metropolitan Institute of Medical Science, Tokyo, Japan. ${ }^{2}$ Nagoya University Graduate School \\ of Medicine, Nagoya, Japan ${ }^{3}$ Fukuoka University Faculty of Medicine, Fukuoka, Japan
}

Although C57BL/6J (B6) mice develop age-related hearing loss (AHL), B6-Chr $17^{\mathrm{MSM} / \mathrm{Ms}}$ consomic mice, where chromosome 17 is substituted with its counterpart from MSM/Ms (MSM) mice into the B6 genetic background, show resistance to AHL. We hypothesized that high expression of the leucine rich repeat containing 30 gene (Lrrc30) in B6-Chr $17^{\mathrm{MSM}}$ mice was a factor suppressing the onset of AHL because the expression of Lrrc30 in MSM mice was significantly higher than that of B6 mice. To confirm this hypothesis, we performed two reverse genetic approaches. First, we produced Lrrc30 knockout (KO) mice in the B6-Chr17 ${ }^{\mathrm{MSM}}$ genetic background and measured their hearing levels. However, Lrrc30
KO mice showed normal hearing levels. Next, we produced bacterial artificial chromosome transgenic (BAC-tg) mice by microinjection of an MSM-derived BAC clone including full-length Lrrc30. The mean expression levels of Lrrc30 were approximately 4-fold higher in BAC-tg compared with B6J mice. We measured hearing levels of BAC-tg mice and confirmed that the hearing loss at high frequency tones in B6 mice was improved in BAC-tg mice. These results suggest that Lrrc30 is not essential in the hearing ability of mice, but increased expression of Lrrc30 suppresses the onset of AHL. 


\title{
$0-25$ \\ Analysis of mice deficient in a placenta-specific, paternally expressed gene, Sfmbt 2 .
}

\author{
$\bigcirc$ Kimiko Inoue, Michiko Hirose, Ayumi Hasegawa, Keiji Mochida, Atsuo Ogura
}

Bioresource Center, RIKEN, Tsukuba, Japan

The Sfmbt2 gene is expressed in placentas and known as a rodent-specific imprint gene. A previous report indicated that Sfmbt2 paternal knockout mice showed embryonic lethality because of reduced placenta. However, its function during placentation is still unknown. In this study, we produced Sfmbt2-deficient mice to uncover their function. We designed gRNAs in the Sfmbt2 gene and transferred them into fertilized embryos with Cas9 protein or mRNA. These embryos were transferred into pseudopregnant females on the next day and we obtained three heterozygous mutant neonates. A weaned female mouse was mated with a wildtype male and we confirmed the mutation was inherited into offspring. However, paternal allele knockout mice showed no reduced placental development. We also found that there are no null knockout fetuses by mating of heterozygous parents. This result showed that Sfmbt2 gene is essential for post-implantation development in mice.

\section{0-26 Sperm-borne phospholipase C zeta-1 ensures monospermic fertilization in mice}

\author{
$\bigcirc$ Yuhkoh Satouh ${ }^{1}$, Kaori Nozawa ${ }^{1,2,3}$, Masahito Ikawa ${ }^{1,3,4}$ \\ ${ }^{1}$ Research Institute for Microbial Diseases, Osaka University, Suita, Osaka, Japan. ${ }^{2}$ Baylor College of Medicine, Houston, USA. ${ }^{3}$ Graduate \\ School of Medicine, Osaka University, Suita, Osaka, Japan. ${ }^{4}$ The Institute of Medical Science, The University of Tokyo, Tokyo, Japan.
}

In mammal, entry of sperm into oocytes triggers the $\mathrm{Ca}^{2+}$ oscillations that initiate resumption of the meiotic cell cycle. In this study, we identified phospholipase C zeta 1 (PLCzl) in sperm head as the sperm-borne oocyte activation factor (SOAF). We generated Plczl knockout (KO) mice by CRISPR/Cas 9 system, and found that the $\mathrm{KO}$ spermatozoa completely failed to induce $\mathrm{Ca}^{2+}$ changes in intracytoplasmic sperm injection (ICSI), but induced atypical patterns of $\mathrm{Ca}^{2+}$ changes in normal fertilizations. Furthermore, most of the oocytes fertilized by KO spermatozoa stop their development at the 1 cell stage because of oocyte activation failure or polyspermy. As a result of examination of two major systems for block of polyspermy, zona pellucida block to polyspermy (ZPBP) and plasma membrane block to polyspermy (PMBP), both of them were shown to delay in the oocytes fertilized by KO spermatozoa. Finally, we obtained healthy pups from male mice carrying human infertile PLCZ1 mutation by single sperm ICSI supplemented with PlcZl mRNA injection. These results suggest that PLCzl is the longsought SOAF that can ensure oocyte activation for monospermic fertilization, and further, that mammalian spermatozoa have an oocyte activation mechanism independent from PLCzl. 


\title{
RSPH6A is essential for sperm flagellum formation and male
} fertility in mice

\author{
$\bigcirc$ Haruhiko Miyata ${ }^{1}$, Ferheen Abbasi ${ }^{1,2}$, Keisuke Shimada ${ }^{1}$, Takafumi Matsumura ${ }^{1,3}$, \\ Akane Morohoshi ${ }^{1,2}$, Masahito Ikawa ${ }^{1,2,3}$ \\ ${ }^{1}$ Research Institute for Microbial Diseases, Osaka University, Suita, Japan ${ }^{2}$ Graduate School of Medicine, Osaka \\ University, Suita, Japan ${ }^{3}$ Graduate School of Pharmaceutical Sciences, Osaka University, Suita, Japan
}

The radial spokes found within cilia and flagella axonemes are $\mathrm{T}$-shaped protein complexes that have a rod-like structure attached to individual outer microtubule doublets and project towards the central pair of microtubules. While the radial spoke proteins have been extensively studied in the unicellular flagellate Chlamydomonas, their function in mammals has yet to be fully elucidated. Mutant Chlamydomonas that lack some or all of the components of this complex have immotile flagella. Abnormal cilia motility and defective axonemal structures in humans cause several syndromic diseases termed ciliopathies, such as Primary Ciliary Dyskinesia. In several of these syndromic ciliopathies, male infertility due to immotile spermatozoa occurs. RSPH6A is a radial spoke protein located in the spokehead and is evolutionarily conserved from Chlamydomonas to humans. Here, we generated Rsph6a knockout mice using the CRISPR/ Cas9 system to clarify the function of Rsph6a. Homozygous mutant mice were viable, but no offspring were obtained from these mice when mated to wild-type females. Further analysis showed that spermatozoa obtained from the cauda epididymis possessed short tails and were immotile. These results indicate that RSPH6A is essential for sperm flagellum formation and male fertility in mice.

\section{0-28}

\section{Heterochromatin Protein 1 (HP1) represses the neuron specific} genes in neural stem cells

\author{
$\bigcirc$ Chie Naruse ${ }^{1}$, Toru Yoshihara ${ }^{1}$, Kanae $\mathrm{Abe}^{2}$, Tomoaki Kato ${ }^{3}$, Takumi Nishiuchi ${ }^{3}$, \\ Masahide Asano $^{1}$ \\ ${ }^{1}$ Institute of Laboratory Animals, Graduate School of Medicine, Kyoto University, Kyoto, Japan ${ }^{2}$ Institute for Experimental Animals, Advanced Science Research \\ Center, Kanazawa University, Kanazawa, Japan ${ }^{3}$ Institute for Gene Research, Advanced Science Research Center, Kanazawa University, Kanazawa, Japan
}

Heterochromatin Protein 1 (HP1) family members are components of constitutive and facultative heterochromatin in eukaryotic cells. HPl proteins bind to di- and tri-methylated histone H3 lysine 9 (H3K9me2/3). We analyzed HP1deficient embryonic brains and neurospheres derived from embryonic day 14.5 embryos to reveal the functions of HPl in neural tissues. HPl-deficient neurospheres had a tendency toward differentiation. Expression array and qRT-PCR analyses revealed several genes expressed in differentiated neural cells were upregulated in HPl-deficient neurospheres compared to wild-type neurospheres. Levels of tri-methylated histones were reduced around the transcription start sites of these genes in HPldeficient neurospheres compared to wild-type neurospheres. The levels of histone demethylases were increased at the promoter sites of these genes in HPl-deficient neurospheres, suggesting that HPl in neurospheres was required for interruption of recruitment of the histone demethylases at the promoter sites of differentiated cell-specific genes to repress expression of these genes in the neural precursor cells. 


\title{
$0-29$ \\ Maternal deficit in methionine metabolism alters behavioral traits and gene expression of progenies
}

\author{
Tamio Fuuse ${ }^{1}$, Takashi Kohda ${ }^{2}$, Tomoko Kushida ${ }^{1}$, Ikuko Yamada ${ }^{1}$, Ikuo Miura ${ }^{1}$, \\ Kimio Kobayashi ${ }^{1}$, Fumitoshi Ishino ${ }^{2}$, Shigeharu Wakana ${ }^{1}$ \\ ${ }^{1}$ Japan mouse clinic, RIKEN BRC, Tsukuba, Japan ${ }^{2}$ Dept. of Epigenetics, Tokyo Med. \& Dent. Univ., \\ Tokyo, Japan
}

Developmental Origins of Health and Disease (DOHaD) is the concept that in utero experiences reprogram susceptibility to adult phenotypes. The aim of our study is to validate the DOHaD hypothesis in the behavioral traits using mouse models. In the previous study, we provided protein restricted (PR) diet and folate supplemented $\mathrm{PR}$ diet to mothers in order to manipulate fetal environments. In the study, PR and controldiet progenies showed moderate differences in behavioral traits. Changes were also detected in gene expression and genomic methylation in the brain. Part of these behavioral, gene expression, and DNA methylation changes were cancel by folate supplementation. The folate is metabolized in one carbon metabolism (OCM). Therefore, we focused on the OCM in the present study. The OCM is consisted of methionine cycle and folate cycle. The folate cycle is involved in DNA synthesis and methionine cycle is involved in DNA methylation by donating methyl group. We produced wild type progeny from mutant females of key genes of the OCM such as Mat2a by using in vitro fertilization technique. The progenies obtained from heterozygote of Mat2a KO mice exhibited increased spontaneous locomotor activity in the behavioral phenotyping. In addition, we measured examined gene-expression analyses in several brain regions.

\section{0-30 Early postnatal vocalizations predict sociability and spatial memory in C57BL/6J mice}

\author{
Kaichi Yoshizaki ${ }^{1}$, Kohei Koike ${ }^{2,3}$, Ryuichi Kimura ${ }^{2}$, Noriko Osumi² \\ 'Department of Pathology, Institute for Developmental Research, Aichi Human Service Center, Aichi, Japan 2Department of Develoomental Neuroscience, United Centers for Advanced Research and Trans lational Medicine, \\ Tohoku University Graduate School of Medicine, Sendai, Japan ${ }^{3}$ Department of Physiology, Center for Integrative Physilogy and Molecular Medicine, Saarland University School of Medicine, Homburg, Germany
}

The understanding of individual diversity and its link to brain functions is a fundamental issue in neurobiology. Studies in mice have mainly focused on the investigation of behavior traits in adulthood, whereas longitudinal analyses are largely uninvestigated. Here we have conducted systematic behavior tests in individual mice (C57BL6/J, male), comparing phenotypes at early postnatal stages and in adulthood. Each animal showed different scores in individual behavior tests. However, we observed an inverse correlation between repetitive behavior in the Morris water maze test and sociability in the 3-chamber social interaction test; an increase in repetitive behaviors was associated with poor sociability. In longitudinal analyses, the emission of ultrasonic vocalization during maternal separation at postnatal day 6 in pups was correlated positively with sociability and negatively with spatial memory. Our results show a possibility that individual differences in communication between pups and their mother in infancy is a predictive indicator for sociability and cognitive performance as an adult. 


\title{
0-31 Functional analysis of $P h f 24$ gene in rats
}

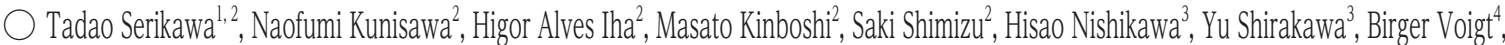
Satoshi Nakanishi', Takashi Kuramoto ${ }^{4}$, Takehito Kaneko, ${ }^{4,5}$, Takashi Yamamoto $0^{6}$, Tomoji Mashimo ${ }^{4,7}$, Masashi Sasa ${ }^{1,8}$, Yukihiro Ohno ${ }^{2}$

${ }^{1}$ Kyoto Disease Model Institute, Kyoto, Japan ${ }^{2}$ Osaka University of Pharmacological Sciences, Takatsuki, Japan ${ }^{3}$ KAC Co. Ltd, Kyoto, Japan ${ }^{4}$ Kyoto University, Kyoto, Japan ${ }^{5}$ Iwate University, Morioko, Japan ${ }^{6}$ Hiroshima University, Hiroshima, Japan ${ }^{7}$ Osaka University, Suita, Japan ${ }^{8}$ Nagisa Clinic, Hirakata, Japan

Phf24 was identified as a candidate gene involved in generalized tonic-clonic seizures (GTCS) in Noda Epileptic Rat (NER) (Kuramoto et al. Behav Genet 2017). To study the function of Phf24, we have developed Phf24-KO rats and investigated changes in behavior and gene expression. F344-Phf24-KO (Phf24-KO) rats were generated by applying the TALEN technology. Emotional behavior test, PTZ-induced convulsion test, PTZ-kindling test, elevated plus-maze test, and Morris water maze test were conducted. The data obtained were compared to the values of the control rats (F344/Stm or F344/NSlc). Phf24$\mathrm{KO}$ rats showed different behavioral phenotypes in several tests. RNA samples were prepared from the amygdala tissues of Phf24-KO and F344/Stm rats, respectively. DNA microarray analysis was performed by Takara Bio. 32 downregulated genes with a fold change $<0.5$ and 58 up-regulated genes with a fold change $>2.0$ were identified in Phf24-KO. The present study supports that $P h f 24$ is a candidate gene for GTCS in NER. We evaluated that Phf24-KO rat will be a valuable model to study Phf24 function.

\section{0-32 Histological analysis of deficient mice in $\beta-1,4$ galactosyltransferases}

\author{
$\bigcirc$ Toru Yoshihara ${ }^{1,2}$, Hiroyuki Satake ${ }^{2}$, Nozomu Okino ${ }^{3}$, Toshihisa Hatta ${ }^{4}$, Hiroki Otani ${ }^{5}$, \\ Kazushi Sugihara ${ }^{1,2}$, Koichi Furukawa ${ }^{6,7}$, Makoto Ito $^{3}$, Masahide Asano ${ }^{1,2}$ \\ Inst. of Lab. Anim., Grad. Sch. of Med., Kyoto Univ., Kyoto, Japan Adv. Sci. Res. Ctr., Kanazawa Univ., Kanazawa, Japan ${ }^{3}$ Grad. Sch. Bioresource Bioenvtl. Sci., Kyushu Univ., Hukuoka, Japan \\ ${ }^{4}$ Kanazawa Medical Univ., Uchinada, Japan ${ }^{5}$ Fac. Med., Shimane Univ., Izumo, Japan ${ }^{6}$ Grad. Sch. Med., Nagoya Univ., Nagoya, Japan ${ }^{7}$ Chubu Univ. Col. Life, Health Sci., Kasugai, Japan
}

$\beta$-1,4-galactosyltransferase ( $\beta$ 4GalT) -5 and $\beta$ 4GalT-6 act as lactosylceramide (LacCer) synthases in several mouse organs (Nishie et al., 2010, Tokuda et al., 2013). Here, we generated conditional $\beta$ 4GalT-5 knockout mice using Nestin-Cre mice crossed with $\beta 4$ GalT-6 KO mice to generate $\beta 4 \mathrm{GalT}-5$ and -6 double $\mathrm{KO}$ (DKO) mice in the central nervous system. LacCer synthase activity and brain gangliosides were disappeared in the brain of DKO mice. The DKO mice showed growth retardation and motor deficits and died by 4 weeks of age. Histological analyses showed that myelin-associated proteins were rarely found localized in axons and axonal/ myelin formation were remarkably impaired. Neuronal cells differentiated from neurospheres of the DKO mice showed disturbed neurite outgrowth and branch formation. This might be because the neurospheres from DKO mice were unable to interact with laminin owing to a lack of gangliosides. Furthermore, the neurons were immature and perineuronal nets were poorly formed in the cerebral cortices of DKO mice. 


\title{
0-33 Mutation analysis of mouse colon organoids at an early stage of PhIP-induced carcinogenesis
}

\author{
Toshio Imai $^{1}$, Mie Naruse ${ }^{1}$, Masako Ochiai ${ }^{1}$, Yoshitaka Hippo ${ }^{2}$ \\ ${ }^{1}$ Ctr Anim Div, Natl Cancer Ctr Res Inst, Tokyo, Japan ${ }^{2}$ Dept Mol Carcinogenesis, Chiba Cancer Ctr \\ Res Inst, Chiba, Japan
}

The mechanisms of multistep carcinogenesis by long-time treatment of chemicals in vivo have not been comprehensively understood. We reported that subcutaneously injected mouse colon-derived organoids, to which Kras activation, Apc, p53 and/or Pten knockdown have been introduced in vitro, showed tumorigenicity. In the present study, analyses for carcinogenic potential and early mutation patterns by a short-time treatment of 2-amino1-methyl-6-phenylimidazo[4,5-b]pyridine (PhIP) was conducted using organoids. [Materials and methods] After introduction of shLuc (control) or shPten followed by PhIP treatment $(0,15,75 \mu$ $\mathrm{M}, \mathrm{x} 3)$, the B6 mouse colon-derived organoids were injected to nude mice. At 44 days after the injection, subcutaneous tissues were histopathologically examined. [Results] Although atypical/invasive lesions and carcinomas were induced in shPten+PhIP 15 and $75 \mu$ M groups, respectively, no tumor formation was observed in shLuc+PhIP groups. A whole exome analysis using DNA isolated from the organoids revealed no mutation in shLuc $+\mathrm{PhIP}$ $15 \mu \mathrm{M}$ and 7 mutations in shPten+PhIP $15 \mu \mathrm{M}$ groups. The mutations were found not in directly carcinogenesis-related genes, but in others, e.g. a protein stability-related gene. [Discussion] The tumorigenesis by shPten+PhIP treatment was considered to be related to increased susceptibilities to PhIP by KI3K-Akt signaling stimulation or genomic instability by Pten knockdown.

\section{0-34 Comparison of healing effect of catechin and L-theanine against gastrointestinal ulcer}

\author{
Masahiko Fujisawa, Yuri Tachibana, Sayaka Hanazono, Yoji Hakamata \\ School of Veterinary Nursing and Technology, Nippon Veterinary \& Life Science University, Tokyo, \\ Japan
}

Background \& Aims: Efficacy and safety of tea components as dietary supplement has been assessed in recent years. Epigallocatechin gallate (EGCG) is the abundant among the four gallatetype catechins and shows extensive physiological activity. Also L-theanine which is a trace amino acid has a central or hepatic metabolism stimulatory action. However, there are few studies on ulcer research of each component. In this study, the morphologic comparison was examined on the therapeutic effects of duodenal ulcer of both components. Materials \& Methods: C57BL / 6J male mice (average $25 \mathrm{~g}$ ) were used throughout the whole experiment. EGCG and L-theanine (LT, 1, 3, $10 \mathrm{mg} / \mathrm{kg}$, ip) were treated 24-hr before/after treatment of cysteamine (CYS)
(300 mg / kg, po). The duodenum part was sampled up to $4 \mathrm{~cm}$ from the pyloric part, and mucosal injury was assessed by macroscopic observation. Results: Both tea components showed the decreasing trend of ulcer scores was confirmed in post-treatment groups rather than pre-treatment groups. In the LT posttreatment group, a dose-dependent decrease of the score was observed; in particular it was significant in the $3 \mathrm{mg}$ treatment group. Likewise, ulcer scores tended to decrease also in EGCG group, especially the tendency was remarkable in the $10 \mathrm{mg}$ treatment group. Conclusion: Both tea components are presumed to promote the cure of duodenal mucosal disorder induced by cysteamine. 


\section{0-35 \\ The effect of the thawing period on the fertilization ability of sperm frozen using the CARD method}

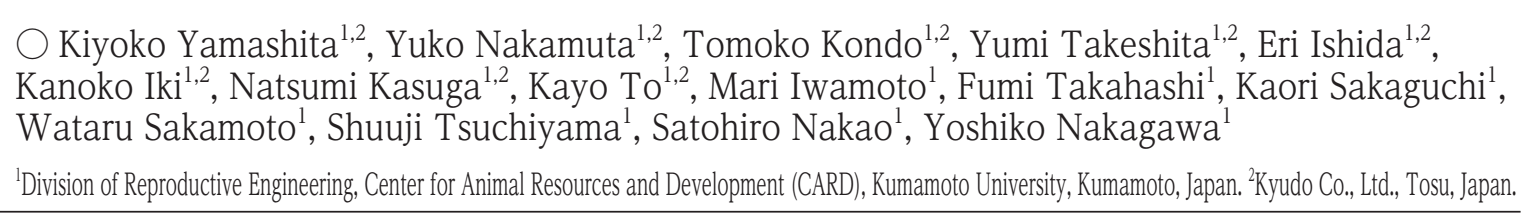

Sperm cryopreservation is a useful method of efficiently archiving genetically engineered mice. In the CARD Mouse Bank, we archived forty thousand straws of cryopreserved sperm derived from genetically engineered mice. To enhance the efficiency of embryo production, we developed a system of sperm cryopreservation and in vitro fertilization which yielded a high fertilization rate. Usually, the cryopreserved sperm were thawed at $37^{\circ} \mathrm{C}$ for 10 minutes in
CARD method. However, the acceptable range of thawing period had not been examined.

In this study, we evaluated the effect of thawing period on the fertilization rate of frozen-thawed sperm. Fertilization rates were highly maintained for 10 minutes. However, longer thawing periods tended to reduce the fertilization rate. In summary, a thawing period of 10 minutes or less may yield high fertilization rates in frozenthawed sperm.

\footnotetext{
$0-36$ Verification of the cold transport of mouse cauda epididymides using various strains of mice

Tomoko Kondo ${ }^{1,2}$, Yuko Nakamuta, ${ }^{1,2}$, Yumi Takeshita ${ }^{1,2}$, Eri Ishida ${ }^{1,2}$, Kanoko Iki, ${ }^{1,2}$, Natsumi Kasuga ${ }^{1,2}$, Kayo To ${ }^{1,2}$, Kiyoko Yamashita ${ }^{1,2}$, Mari Iwamoto', Fumi Takahashi, Kaori Sakaguchi', Shuuji Tsuchiyama ${ }^{1}$, Wataru Sakamoto', Hidetaka Yoshimoto, Satohiro Nakao ${ }^{1}$, Yoshiko Nakagawa ${ }^{1}$, Toru Takeo ${ }^{1}$, Norihiko Shimizu ${ }^{3}$, Chihiro Hino ${ }^{3}$, Naomi Nakagata ${ }^{1}$ ${ }^{1}$ Division of Reproductive Engineering, Center for Animal Resources and Development (CARD), Kumamoto University, Kumamoto, Japan. ${ }^{2}$ Kyudo Co., Ltd., Tosu, Japan. ${ }^{3}$ Center for Advanced Research and Education Asahikawa Medical University, Asahikawa, Japan
}

The cold transport of cauda epididymis enables the transport of genetically engineered mice without the risk of mouse death or escape during transport, the spread of infectious disease, or the violation of animal welfare regulations. Recently, we developed a new technique which maintains the fertility of cold-stored mouse sperm for 10 days at $4^{\circ} \mathrm{C}$. In this study, we evaluated the efficacy of this cold-transport system using various strains of mice. We examined the fertilization and developmental ability of cold-stored sperm collected from cauda epididymides of various strains of mice (BALB/cByJJcl, C3H/HeJJcl, DBA/2JJcl, FVB/ $\mathrm{NJcl}, \mathrm{Jcl}: \mathrm{BDF} 1$ and Jcl:ICR) after their shipment from Asahikawa Medical University to CARD, Kumamoto University. We confirmed the fertility and developmental ability of cold-stored sperm in each of the aforementioned mouse strains. In summary, this system is useful for transporting genetically engineered mice derived from various strains of mice. 

international transportation at $-79^{\circ} \mathrm{C}$

$\bigcirc$ Kanoko Iki ${ }^{1,2}$, Yuko Nakamuta ${ }^{1,2}$, Tomoko Kondo ${ }^{1,2}$, Yumi Takeshita ${ }^{1,2}$, Eri Ishida ${ }^{1,2}$, Natsumi Kasuga $^{1,2}$, Kayo To ${ }^{1,2}$, Kiyoko Yamashita ${ }^{1,2}$, Mari Iwamoto ${ }^{1}$, Fumi Takahashi ${ }^{1}$, Kaori Sakaguchi ${ }^{1}$, Shuuji Tsuchiyama ${ }^{1}$, Wataru Sakamoto ${ }^{1}$, Satohiro Nakao ${ }^{1}$, Yoshiko Nakagawa ${ }^{1}$

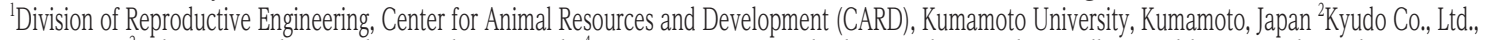
Tosu, Japan ${ }^{3}$ Italian National Research Council, Rome, Italy ${ }^{4}$ Mary Lyon Centre, Medical Research Council Harwell, Oxfordshire, United Kingdom

Cryopreserved sperm can be used for in vitro fertilization (IVF) and embryo transfer (ET) to produce live pups. However, there is a drawback to transporting frozen sperm from the standpoint of shipment containers. In general, a dryshipper is used to maintain the sperm at $-196^{\circ} \mathrm{C}$ during transportation. The container should be returned after shipment, which requires extra work and expense. To overcome this problem, we evaluated the efficacy of using a simple container containing dry ice $\left(-79^{\circ} \mathrm{C}\right)$ for the shipment of cryopreserved mouse sperm. Cryopreserved sperm were transported from MRC Harwell to CARD, where their fertility and developmental ability were examined. Frozen sperm were fertilized via IVF, and live pups derived from the sperm were obtained via ET. In summary, we verified that the transport of cryopreserved mouse sperm using dry ice $\left(-79^{\circ} \mathrm{C}\right)$ instead of liquid nitrogen $\left(-196^{\circ} \mathrm{C}\right)$ could be utilized for the international transport of genetically engineered mice.

\title{
0-38 Vitrification of mouse zygotes; effect of rapid warming
}

\author{
Shinsuke Seki, Keita Basaki, Yukie Komatsu, Yasuyoshi Fukuda, Megumi Yano, \\ Yuhei Matsuo, Takahiro Obata, Yukihisa Matsuda, Kazutoshi Nishijima \\ Bioscience Education and Research Support Center, Akita University, Akita, Japan
}

The findings we reported on the vitrification of mouse oocytes using cryotops is that the survival of mouse oocytes was high even with moderate cooling rate and the vitrification solution containing only a half the usual concentration of cryoprotectants if the warming rate was extremely high. Then, we vitrified mouse zygotes with cryotubes and examined the effect of warming rate and the concentration of cryoprotectants on the survival. Vitrification solutions were EFS20, EFS30, and EFS40, which contained ethylene glycol (20\%, 30\% and 40\% $\mathrm{V} / \mathrm{v}$, respectively), ficoll, and sucrose. After exposure to the EFS solution for 2 min at $23^{\circ}$ C, $5 \mu \mathrm{l}$ of the solution suspended with mouse zygotes in a cryotube was vitrified by direct immersion into liquid nitrogen. They were warmed, a) by holding at $23^{\circ} \mathrm{C}$ for $3 \min \left(34^{\circ} \mathrm{C} /\right.$ min), b) by adding $1 \mathrm{ml}$ solution containing 0.5 $\mathrm{M}$ sucrose (sucrose solution) at $23^{\circ} \mathrm{C}\left(4,600^{\circ} \mathrm{C} /\right.$ $\mathrm{min}$ ), or c) by adding $1 \mathrm{ml}$ sucrose solution at 37 ${ }^{\circ} \mathrm{C}\left(6,600^{\circ} \mathrm{C} / \mathrm{min}\right)$. With EFS40, the survival was low regardless of the warming rate. With EFS30 and EFS20, the survival was low if the warming rate was low. With EFS20 and rapid warming, almost all the zygotes developed to blastocysts in vitro. In conclusion, we developed a vitrification method for mouse zygotes using cryotubes by rapid warming. We believe that this system will be applied to other species (rat or rabbit) besides mice. 


\title{
0-39 Ultra-superovulation for the genetically engineered mice production.
}

\author{
$\bigcirc$ Hitoshi Miyachi ${ }^{1}$, Satsuki Kitano ${ }^{1}$, Katsuhiko Itoh ${ }^{2}$, Naomi Nakagata ${ }^{3}$, Koichi Ikuta ${ }^{1}$ \\ ${ }^{1}$ Institute for Frontier Life and Medical Sciences, Kyoto University, Kyoto, Japan ${ }^{2}$ Department of Clinical Molecular Biology, Graduate School of \\ Medicine, Kyoto University, Kyoto, Japan ${ }^{3}$ Center for Animal Resources and Development, Kumamoto University, Kumamoto, Japan
}

Recently, a novel ultra-superovaluation technique has been developed by using anti-inhibin antisera in mice. This technique is very practical, since we can obtain the greater number of oocytes by this technique than that by the conventional one. It is also a good technique from the viewpoint of animal welfare, as it can reduce the number of mice sacrificed. Furthermore, ultrasuperovaluation technique have been shown to be able to use for down-stream applications, such as in vitro fertilization and etc, as stably as the conventional one.In the present study, we compared the efficiency to generate genetically engineered mice using oocytes obtained by the ultra-superovaluation with that by conventional superovaluation. We purchased HyperOva or Serotropin for animals, from Kyudo Inc. or Asuka Animal Helth Inc., and used them for the induction of ultra-superovaluation or conventional superovaluation, respectively. All embryos were cryopreservated in liquid nitrogen and used for experiments after thawing.Although the number of experiments was small, we did not observe significant difference in the efficiency of genetically engineered mice ( $\mathrm{Tg}, \mathrm{KO}$ and CRISPR) between the oocytes obtained by ultrasuperovaluation and those by conventional superovaluation.

\section{0-40 An efficient system for producing homozygous GEM strains using reproductive technology}

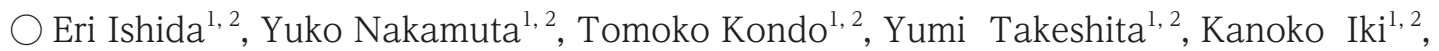
Natsumi Kasuga ${ }^{1,2}$, Kayo To ${ }^{1,2}$, Kiyoko Yamashita ${ }^{1,2}$, Naoko Nakamura ${ }^{3}$, Masami Kawabe ${ }^{3}$, Mari Iwamoto $^{1}$, Fumi Takahashi ${ }^{1}$, Kaori Sakaguchi ${ }^{1}$, Wataru Sakamoto ${ }^{1}$, Shuuji Tsuchiyama

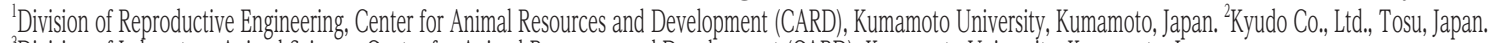
${ }^{3}$ Division of Laboratory Animal Science, Center for Animal Resources and Development (CARD), Kumamoto University, Kumamoto, Japan.

In general, it takes over a year to expand a mouse colony via natural mating using a small number of genetically engineered homozygous mice. In this study, we evaluated the efficacy of using mouse reproductive technology to expand a colony and to produce cryopreserved embryos for backup. Firstly, we produced over thirty mice pups via in vitro fertilization, carried out using a cauda epididymis collected from an adult male mouse and three immature female mice, and embryo transfer. Almost all of the mice produced were used to expand the colony, but we used five of the female pups and the cauda epididymis of one male mouse for in vitro fertilization and embryo cryopreservation for backup use. As a result, we were able to produce enough pups for breeding and enough cryopreserved embryos for archiving in four months. 


\title{
$0-41$
}

Inventory control of cryopreserved mouse embryos in the CARD Mouse Bank

Kayo To ${ }^{1}$, Yuko Nakamuta ${ }^{1,2}$, Tomoko Kondo ${ }^{1,2}$, Yumi Takeshita ${ }^{1,2}$, Eri Ishida ${ }^{1,2}$, Kanoko Iki ${ }^{1,}$ ${ }^{2}$, Natsumi Kasuga ${ }^{1,2}$, Kiyoko Yamashita ${ }^{1,2}$, Mari Iwamoto ${ }^{1}$, Fumi Takahashi ${ }^{1}$, Kaori Sakaguchi ${ }^{1}$, Shuuji Tsuchiyama ${ }^{1}$, Wataru Sakamoto ${ }^{1}$, Satohiro Nakao', Yoshiko Nakagawa ${ }^{1}$

'Division of Reproductive Engineering, Center for Animal Resources and Development (CARD), Kumamoto University, Kumamoto, Japan. ${ }^{2}$ Kyudo Co., Ltd., Tosu, Japan.

In the CARD mouse bank, we record basic information concerning the archived mouse lines, results of breeding using reproductive technology, and inventory records of cryopreserved sperm and embryos so as to enable us to quickly supply mouse lines when a customer orders them. For each mouse line, we store over five tubes of cryopreserved embryos (sixty embryos/ tube) and ten straws of cryopreserved sperm. The cryopreserved embryos and sperm are replenished after samples are supplied. To ensure the smooth supply of mouse lines against a backdrop of increased demand, we particularly need to develop an efficient system of replenishing cryopreserved mouse embryos. In this study, we evaluated the efficacy of in vitro fertilization between the cryopreserved sperm of genetically engineered mice and oocytes derived from ultra-superovulation, with the goal of increasing the cryopreserved mouse embryo archiving efficiency. We were able to produce a sufficient number of embryos via in vitro fertilization between the aforementioned cryopreserved sperm and oocytes. The produced embryos survived vitrification and warming, and subsequently developed to live pups via embryo transfer.

\section{0-42 Lipid raft dynamics linked to sperm competency for fertilization in mice}

\author{
$\bigcirc$ Hitomi Watanabe, Rie Takeda, Keiji Hirota, Gen Kondoh \\ Institute for Frontier Life and Medical Sciences, Kyoto University, Kyoto, Japan
}

Mammalian sperm acquires fertilization ability after several maturation processes, particularly within the female reproductive tract. Here, we show that lipid raft movement is fundamental for sperm to be competent for fertilization by comparing the sperm maturation process of two mouse inbred strains, C57BL/6 and $\mathrm{BALB} / \mathrm{c}$. We found that ganglioside GMI movement was exclusively reduced in BALB/ c compared with C57BL/6 among other examined sperm maturation parameters, such as glycosylphosphatidylinositol (GPI)-anchored protein (GPI-AP) release, sperm migration to the oviduct, cholesterol efflux, protein tyrosine phosphorylation and acrosome reaction, and was strongly linked to sperm fertility phenotype. The relationship between GMl movement and in vitro fertilization ability was confirmed in other mouse strains, suggesting that lipid raft movement is one of the important steps for completing the sperm maturation process. 

genome editing animals

\author{
$\bigcirc$ Takehito Kaneko ${ }^{1,2}$, Yuki Nakagawa ${ }^{1}$ \\ ${ }^{1}$ Graduate School of Arts and Science, Iwate University, Iwate, Japan ${ }^{2}$ Department of Chemistry and \\ Biological Sciences, Faculty of Science and Engineering, Iwate University, Iwate, Japan
}

Electroporation method (TAKE method) has been developed for generation of genome editing animals by Kaneko et al. In this study, we examined the improvement of easy and efficient TAKE method. C57BL/6J x ICR mouse embryos were used in this study. Tyrosinase gene was used for targeted gene. Cas9 nuclease and dualRNA were electroporated into embryos using NEPA21 with $1 \mathrm{~mm}$ gap electrode. Embryos were then transferred to pseudopregnant females. Some offspring showed modification of targeted gene. This study demonstrated that the electroporation of Cas9 nuclease and dualRNA into embryos using NEPA21 with $1 \mathrm{~mm}$ gap electrode is low cost and more efficient method for generation of genome editing animals.

\title{
0-44 Optimizing the generation of genetically engineered mice with CRISPR/Cas9 system in zygotes.
}

\author{
$\bigcirc$ Hiroshi Kiyonari, ${ }^{1,2}$, Takaya $\mathrm{Abe}^{1,2}$, Ken-ichi Inoue ${ }^{1}$, Miho Kihara ${ }^{1}$, Karin Akiyama ${ }^{1}$, \\ Mayo Shigeta ${ }^{1}$, Yasuhide Furuta ${ }^{1,2}$ \\ ${ }^{1}$ Animal Resource Development Unit, RIKEN CLST, Kobe, Japan ${ }^{2}$ Genetic Engineering Team, RIKEN \\ CLST, Kobe, Japan
}

The CRISPR/Cas9 system allows for generation of genetically engineered mice by microinjection into zygotes, and a number of approaches to enhance the efficiency of genome modifications have been reported recently. However, there appears to be a room for improvements on the technologies to insert specific sequences and gene cassettes in targeted manners. Here we report our current approaches for the generation of designed-null alleles with insertion of termination codons or conditional (floxed) alleles with ssODN, as well as cassette knock-in alleles. For generating knock-in mice efficiently, we attempted to optimize four previously described methods: 1) homologous recombination (HR),
2) that using a long single strand DNA as a donor (lssDNA), 3) the PITCh system, and 4) Homology-Mediated End Joining (HMEJ). The ROSA26 locus was used as a model site for the insertion of a GFP expression cassette. Initially, Cas 9 mRNA was used for microinjection of about 200 zygotes per condition, yielding GFP knock-in embryos only by the lssDNA method. On the other hand, GFP knock-in embryos were obtained more efficiently with a combination of Cas9 mRNA and protein in all methods. In this presentation, as well as knock-in mice, we introduce the usefulness of designed-null, floxed mice generative methods and electroporation method with some of the latest data. 


\title{
Delivery style of Cas9 nuclease during pronuclear injection influences mutation mosaicism in rats
}

\author{
Teppei Goto ${ }^{1}$, Kyoko Yogo ${ }^{1}$, Shinichi Hochi², Masumi Hirabayashi ${ }^{1}$ \\ ${ }^{1}$ National Institute for Physiological Sciences, Aichi, Japan ${ }^{2}$ Shinshu University, Nagano, Japan
}

The CRISPR/Cas9 system often causes genotypic mosaics in the founder mutant animals. This study aimed to investigate the effect of delivery style of Cas9 nuclease during pronuclear microinjection on the incidence of mutation mosaicism in rat blastocysts. Pronuclear-stage zygotes from superovulated Crlj:WI rats were microinjected with guide RNA designed for Foxn 1 and one of the three Cas9 nuclease styles (all-in-one vector pX330, mRNA, or nuclease protein), and were allowed to develop into either 2-cell stage in vitro or blastocyst-stage in vivo. In pX330, mRNA and protein groups, successful genome editing was confirmed at 0, 0 and $10 \%$ of 1 -cell zygotes and 0, 23 and $75 \%$ of 2-cell embryos, respectively. Blastocyst harvest was higher in mRNA group (47\%) than pX330 and protein groups (5 and 14\%, respectively). Genotype of the blastocyst was classified into wildtype, heterozygous mutant, hemi-homo mutant, homozygous mutant, or mosaic, based on the sequences of 10 TA cloning plasmids. Proportions of mosaic blastocysts were 75, 45 and $9 \%$ in pX330, mRNA and protein groups, respectively. In contrast, proportions of hemihomo plus homozygous mutant blastocysts were 25,28 and $82 \%$ in pX330, mRNA and protein groups, respectively. Taken together, homozygous mutant founder rats are more likely generated by the CRISPR/Cas9 system when the Cas9 is delivered into pronucleus in a form of nuclease protein rather than plasmid DNA or mRNA.

\section{$0-46$ \\ Correlation of Cas9 expression and induced mutation pattern in mouse embryos}

\author{
$\bigcirc$ Kazuto Yoshimi $^{1,2}$, Sakura Nakatani ${ }^{2}$, Yoshihiro Uno ${ }^{2}$, Yuko Kotani ${ }^{2}$, Yayoi Kunihiro ${ }^{2}$, \\ Yuko Yamauchi' ${ }^{2}$, Tomoji Mashimo, ${ }^{1,2}$ \\ ${ }^{1}$ Genome Editing Research and Development Center, Graduate School of Medicine, Osaka University, Osaka, \\ Japan ${ }^{2}$ Institute of Experimental Animal Sciences, Graduate School of Medicine, Osaka University, Osaka, Japan
}

Genome editing technologies enabled us for a high-throughput, high-efficiency and low-cost production of genetically modified animals. We have established many knockout and knockin mice and rats with CRISPR-Cas9. However, mosaic mutations or no targeted mutations can be detected at several target sites, which makes difficult to establish the mutant strains in mice and rats. In this study, we visualized sequential expression of Cas9 with P2A peptide and GFP protein in mouse embryos and analyzed the relationship between the Cas9 expression level and Cas9-induced mutations at Tyr gene.
Fluorescence observation in mouse embryos at 2-cell stage revealed that Cas9 expression level were widely varied even the same experiment. Genetic analysis showed that there was no correlation between knockout efficiency at Tyr gene and Cas9 expression pattern, while embryos highly expressing Cas9 significantly carried homozygous mutation at the target site. These results suggested that a visualization of Cas9 expression can be a good selection marker for homozygously mutated embryos before the transplantation. 


\title{
0-47 Electroporation-mediated genome editing in mouse zygotes created by IVF via ultra-superovulation
}

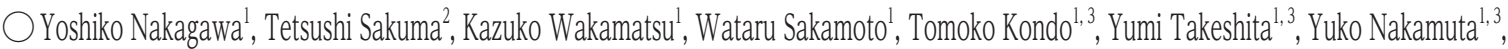 \\ Eri Ishida ${ }^{1,3}$, Yamashita Kiyoko ${ }^{1,3}$, Kanoko Iki ${ }^{1,3}$, Kayo Tou ${ }^{1,3}$, Shuuji Tsuchiyama', Toru Takeo ${ }^{1}$, Takashi Yamamoto ${ }^{2}$, Naomi Nakagata ${ }^{1}$ \\ ${ }^{1}$ Division of Reproductive Engineering, Center for Animal Resources and Development (CARD), Kumamoto University, Kumamoto, Japan. ${ }^{2}$ Department of \\ Mathematical and Life Sciences, Graduate School of Science, Hiroshima University, Hiroshima, Japan. ${ }^{3}$ Kyudo Co., Ltd., Tosu, Japan.
}

We have so far demonstrated the applicability of ultra-superovulation, in vitro fertilization (IVF), and vitrification/warming of zygotes in microinjection-mediated mouse genome editing. On the other hand, the electroporationmediated method has rapidly been established for simple gene knockout and small precise modifications. Here, we present the updated application examples coupling the three latest technologies: 1) CRISPR-Cas9 ribonucleoprotein as the most convenient genome editing reagent, 2) electroporation as the most effortless delivery method, and 3) cryopreserved zygotes created by IVF via ultra-superovulation as the most animal welfare- and user-friendly strategy. We successfully created gene knockout and knockin mice carrying insertion/deletion mutations and single amino acid substitution respectively, using the streamlined production system of mouse genome editing described above. Owing to its accessibility, robustness, and high efficiency, we believe our updated pipeline will be widely used for future global production of genome edited mice.

\section{0-48 Strain difference in the successful production of $i$-GONAD}

\author{
O Shuji Takabayashi ${ }^{1}$, Takuya Aoshima ${ }^{1}$, Katsuya Kabashima ${ }^{1}$, Masahiro Sato², Masato Ohtsuka ${ }^{3}$ \\ ${ }^{1}$ Laboratory Animal Facilities \& Services, Preeminent Medical Photonics Education \& Research Center, Hamamatsu University School of Medicine, \\ Shizuoka, Japan ${ }^{2}$ Section of Gene Expression Regulation, The Center for Advanced Biomedical Science and Swine Research, Kagoshima University, \\ Kagoshima ${ }^{3}$ Department of Molecular Life Science, Division of Basic Medical Science and Molecular Medicine, Tokai University, Kanagawa
}

The improved genome-editing via oviductal nucleic acids delivery (i-GONAD) was first developed as a method to perform genome editing in situ towards preimplantation embryos present within an oviductal lumen. This method employs intaroviductal instillation of genome editing component and subsequent in vivo electroporation towards an entire oviduct, and therefore does not require any of ex vivo handling of preimplantation embryos that is strictly required for production of genomeedited mice using microinjection and in vitro electroporation. GONAD has been confined to only limited mouse strains such as ICR. In this study, we examined whether this technology is applicable to the other inbred and hybrid mice including C57BL/6, C3H/HeN, DBA2, and B6D2F1. The GONAD using C57BL/6 failed to produce any progeny, although all pregnant females were tested. However, it yielded pups that were successfully genome-edited, when the other strains were subjected to the GONAD. Particularly, the hybrid mice B6D2Fl gave best results, in which all pregnant mice tested had delivered their pups and $95 \%$ of these born pups had mutated phenotypes. 


\title{
Two-step generation of conditional knockout mice via sequential introduction of lox sites
}

\author{
Takuro Horii, Sumiyo Morita, Mika Kimura, Naomi Terawaki, Mihiro Shibutani, \\ Izuho Hatada \\ Biosignal Genome Resource Center, Institute for Molecular and Cellular Regulation, Gunma University, \\ Gunma, Japan
}

Conditional knockout using Cre/lox is essential for functional analysis of genes. CRISPR/Cas in combination with two sets of guide RNAs and a single-stranded oligonucleotide enables simultaneous insertion of two lox sequences. However, this method induces double-strand breaks at two sites on the same chromosome, which causes an undesirable chromosomal deletion and reduces the flanked lox (flox) rate. To solve this problem, we investigated a method that sequentially introduces each lox sequence at the 1-cell and 2-cell embryonic stages, respectively.

We found electroporation is suitable for sequential method because survival rate of sequential electroporation is higher than that of conventional microinjection. Sequential electroporation resulted in a lower chromosomal deletion rate (9-38\% vs. 73-81\%) and a higher flox rate $(\sim 23 \%$ vs. $\sim 8 \%)$ than simultaneous electroporation in blastocyst embryos. Moreover, sequential electroporation led to a higher yield of flox offspring in various gene loci than simultaneous electroporation (floxed/born, 13$50 \%$ vs. 4-7\%). Finally, we directly produced Cre/lox mice containing both the Cre transgene and floxed allele via sequential electroporation using Cre zygotes, and found that 20-25\% of Cre mice had a floxed allele. This accelerated the generation of conditional knockout mice compared with the ordinary method.

\section{0-50 Efficient applications of reproductive and genetic engineering technologies to NOD/SCID mice}

\author{
Narumi Ogonuki ${ }^{1}$, Ayumi Hasegawa ${ }^{1}$, Michiko Hirose ${ }^{1}$, Arata Honda ${ }^{1,4}$, \\ Takashi Hiroyama ${ }^{1}$, Keiji Mochida $^{1}$, Yukio Nakamura ${ }^{1}$, Atsuo Ogura ${ }^{1,2,3}$ \\ ${ }^{1}$ RIKEN BRC, Ibaraki, Japan ${ }^{2}$ University of Tsukuba, Ibaraki, Japan ${ }^{3}$ University of Tokyo, Tokyo, Japan \\ ${ }^{4}$ Univaesity of Kyoto
}

Nonobese diabetic/severe combined immunodeficiency (NOD/SCID) mice are extensively used as an ideal model for xenotransplantation experiments. To promote the practical use of NOD/SCID mice, the present study was undertaken to devise reproductive and genetic engineering technologies based on improvement of the protocol for superovulation. Female NOD/SCID mice responded to the conventional eCG/hCG superovulation treatment, but more than half (53\%) of the ovulated oocytes were abnormal in shape, typically showing a small or fragmented ooplasm. When their estrous cycle was synchronized with progesterone and followed by treatment with anti-inhibin serum instead of eCG, 65 oocytes per female were obtained with 94\% normality. The oocytes thus collected showed a normal fertilization rate in vitro (86\%) and 67\% of them developed into offspring after embryo transfer. We confirmed that lentivirus-mediated transgenesis and CRISPR/Cas9-mediated geneknockout could be practically performed using embryos generated by our superovulation protocol. Intracytoplasmic sperm injection was also possible by slightly activating oocytes prior to injection. Thus, our new superovulation protocol makes the NOD/SCID strain one of the strains accessible to reproductive and genetic engineering technologies and would promote its efficient use in biomedical researches. 

treated royal jelly

\author{
$\bigcirc$ Hyejung $\mathrm{Gu}^{1}$, Jungkee Kwon ${ }^{2}$ \\ ${ }^{1}$ Lab. Animal Lab. College of Vet. Med., Chonbuk Nat. Uni., Korea ${ }^{2}$ Lab. Animal Lab., College of Vet. \\ Med., Chonbuk Nat. Uni., Korea
}

Royal jelly (RJ) has been reported to various pharmacological activities. In this study, we investigated the immune enhancement effect of Enzyme-treated royal jelly (ERJ).ERJ showed a significant increase in total free amino acid content with the removal of allergen proteins. The DPPH assay showed about $80 \%$ of ascorbic acid at same concentration. On in vitro tests, ERJ showed macrophage proliferation and protection against lipopolysaccharide (LPS)induced stress. Moreover, ERJ showe the reduction of intracellular reactive oxidative species and nitric oxide production in LPStreated macrophages. ERJ significantly increased the activity of the antioxidant enzyme superoxide dismutase and the level of the antioxidant glutathione in a dose-dependent manner. On in vivo tests, we investigated the antiinflammatory and immunomodulatory effects. The mice fed ERJ for four weeks prior to LPS stimulation exhibited significantly reduced levels of tumor necrosis factor-alpha, interleukins-1, $6,10,12$, and interferon-gamma compared to control mice. In addition, ERJ significantly increased the proliferation of B-lymphocytes and T-lymphocytes, as well as the activity of natural killer cells in a dose-dependent manner. In most data, ERJ showed similar or better anti-oxidant, -inflammatory and immune effects than RJ at the same concentration. These findings indicate that ERJ can be developed as a potential food material for enhancing immune system.

\title{
0-52 Immunomodulating effect of beta-tricalcium phosphate and its potential as a vaccine adjuvant
}

\author{
$\bigcirc$ Kouji Maruyama ${ }^{1}$, Sachiko Tai ${ }^{1}$, Hidee Ishii ${ }^{1}$, Kasumi Shimono ${ }^{1}$, Jing-Yang Cheng ${ }^{2}$, \\ Takatomo Sato $^{2}$, Vincent Zangiacomi ${ }^{1}$, Tetsji Hosono ${ }^{3}$, Ken Yamaguchi ${ }^{4}$ \\ ${ }^{1}$ Shizuoka Cancer Center Research Institute ${ }^{2}$ Advanced Analysis Technology Department, Olympus ${ }^{3}$ Laboratory of \\ Medicinal Microbiology, Yokohama College of Pharmacy ${ }^{4}$ Shizuoka Cancer Center Hospital and Research Institute
}

Beta-tricalcium phosphate ( $\beta$-TCP) has been used for bone substitution in clinical practice. We have reported that $\beta$-TCP has stimulatory effect on the immune system. In the former studies, the following findings have been shown; 1) $\beta$-TCP particles enhanced expression of costimulatory molecules, and induced cytokines and chemokines in mouse dendritic cells (DC) and macrophages $(\mathrm{M} \phi)$. Conditioned medium from DC treated with $\beta$-TCP facilitated migration of splenocytes. In the present study, the effect of $\beta$-TCP on inflammasome, and the potential of $\beta$-TCP as a vaccine adjuvant will be discussed. In brief, $\beta$-TCP particles induced IL-1 $\beta$ production in caspase-dependent manner in murine DC and M $\phi$. In the DC from NLRP3deficient mouse or NLRP3-deficient human monocytic THP-1 cells, $\beta$-TCP failed to induce IL-1 $\beta$ production, suggesting that $\beta$-TCP activates NLRP3 inflammasome. In the E.G7-OVA tumor model, $\beta$-TCP and poly (I:C), a TLR3 ligand, synergistically enhanced antitumor effect of OVA tumor antigen. These findings suggest that the combination of TLR ligand and $\beta$-TCP, a inflammasome activator, could be effective and promising as a vaccine adjuvant. 


\title{
$0-53$ Evaluating the safety of indigo naturalis in patients with ulcerative colitis developing PAH
}

\author{
O Shizuka Uemura ${ }^{1}$, Masaharu Kataoka ${ }^{2}$, Takahiro Hiraide ${ }^{2}$, Toshiaki Teratani ${ }^{2}$, \\ Masahiko Fujisawa ${ }^{1}$, Yoji Hakamata ${ }^{1}$ \\ ${ }^{1}$ Nippon Veterinary and Life Science University, Tokyo, Japan 2Keio University, Tokyo, Japan
}

Recently, Japanese patients with ulcerative colitis taking indigo naturalis (ID), which is a Chinese herbal preparation, developed pulmonary artery hypertension $(\mathrm{PAH})$. To evaluate the safety of ID, we assessed the association between ID consumption and PAH. 5-wks-old F344 male rats were divided into 3 groups: 1) normal diet, CE-2 (ND); 2) ID diet, $40 \mathrm{~g} / \mathrm{kg} / \mathrm{CE}-2$; and 3) IDND diet, ID for 8 wks and ND for 4 wks. Body weight and diet consumption were measured weekly, the right ventricular pressure under anesthesia at 8 and 12 wks after the study was determined invasively, and PAH-related parameters were measured. Next, rats were subcutaneously injected on day 0 with $20 \mathrm{mg} /$ $\mathrm{kg}$ of SU5416 to induce PAH, then administered
ND or ID, and prepared as in the first study at 8 wks. Body weight was significantly lower in the ID group than in the ND group for the entire study period $(\mathrm{P}<0.05)$. Right ventricle enlargement and pulmonary blood vessel medial thickening were significantly greater in the ID group than in the ND group ( $\mathrm{P}<0.05)$. There was also a significant increase in the PAHrelated parameters in the SU5416+ID group compared with those in the ID group after 8 wks $(\mathrm{P}<0.05)$. ID consumption for $12 \mathrm{wks}$ induced moderate PAH in rats; however, discontinuing ID consumption prevented aggravation of PAH. This study suggested that ID consumption has adverse effects on PAH.

\section{0-54 Establishment of GFP/RFP-tagged ES cell and its application for phenotypic analysis}

\author{
Seiya Oura ${ }^{1}$, Haruhiko Miyata ${ }^{1}$, Taichi Noda ${ }^{1}$, Keisuke Shimada ${ }^{1}$, \\ Takashi Matsumura ${ }^{1}$, Akane Morohoshi $^{1}$, Ayako Isotani ${ }^{2}$, masahito ikawa ${ }^{1}$ \\ ${ }^{1}$ Research Institute for Microbial Diseases, Osaka University, Japan. ${ }^{2}$ Department of Biomedical \\ Science, Nara Institute of Science and Technology, Japan
}

CRISPR/Cas9 can introduce biallelic mutations into ES cells (ESCs), and its application with GFP-tagged ESCs enables us to analyze gene functions in chimeric mice. However, when using EGR-G01 ESCs [129S2 x C57BL/6NCr-Tg(CAG/ Acr-EGFP)], analysis of male-fertility related genes becomes difficult because the sperm lose the GFP signal after the acrosome reaction. In this study, we established ESC line from RBGS transgenic mice (B6D2-Tg (CAG/Su9-DsRed2, Acr3-EGFP) RBGS002Osb) whose sperm express GFP in the acrosome and RFP in the flagellar midpiece. We then utilized the ESC when analyzing Hydin, which is reported as a protein related to flagellar motility of sperm in humans.
Phenotypic analysis of Hydin-disrupted sperm is difficult because Hydin-mutated mice die within 3 weeks due to hydrocephalus. We disrupted Hydin in the RBGS-ESCs and generated chimeric mice, which survived and underwent sexual maturation. We then observed spermatozoa of chimeric mice and found red fluorescent (Hydin-/-) sperm possessed short tails and were immotile. When we injected abnormal sperm into oocytes via ICSI, pups were delivered, which indicates that Hydin disruption does not affect genomic integrity. In conclusion, RBGS ES cells are useful for analyzing spermatogenesis and sperm functions in chimeric analysis. 


\title{
0-55 \\ High-throughput mutant mouse production for the International Mouse Phenotyping Consortium
}

\author{
Shinya Ayabe ${ }^{1}$, Kenichi Nakashima ${ }^{2}$, Mizuho Iwama ${ }^{1}$, Toshiaki Nakashiba ${ }^{1}$, Koji Nakade ${ }^{2}$, \\ Maiko Ijuin $^{1}$, Tatsunori Yamamura ${ }^{1}$, Tomomi Hashimoto ${ }^{1}$, Masayo Kadota ${ }^{1}$, Hatsumi Nakata ${ }^{1}$, \\ Takehide Murata $^{2}$, Yuichi Obata ${ }^{2}$, Atsushi Yoshiki ${ }^{1}$ \\ ${ }^{1}$ Experimental Animal Division, RIKEN BioResource Research Center, Tsukuba, Japan ²Gene Engineering Division, RIKEN BioResource Research Center, Tsukuba, Japan
}

RIKEN BioResource Research Center (BRC) participates in the International Mouse Phenotyping Consortium (IMPC) which is currently composed of 18 research institutions. Early efforts at several IMPC centers targeted critical exons to introduce small insertions or deletions (indels) by imprecise non-homologous end-joining (NHEJ) repair of Cas9-mediated double strand breaks. Because of their random nature, indel alleles are difficult to screen and quality control and cannot be standardized. The IMPC shifted its efforts to produce alleles that more closely resembled knockout alleles made in ES cells by using Cas9 to generate deletion alleles. The approach that combines paired gRNAs and Cas9 would be reliable, cost-effective and efficient for producing knockout mice for phenotyping. We extended our approach to electroporation of CRISPR components into zygotes, which can be a convenient and efficient alternative to microinjection for mouse model creation. We also show our recent progress on expanding the allele types of several highvisibility genes in the national scientific community.

\section{0-56 Genome editing technology expands the potential of rat resources.}

Yoshiki Miyasaka ${ }^{1}$, Kosuke Hattori $^{1}$, Yuko Yamauchi ${ }^{1}$, Yuko Kotani $^{1}$, Kanako Shimizu ${ }^{1}$, Yoko Mizuno $^{1}$, Yoshihiro Uno ${ }^{1}$, Kazuto Yoshimi ${ }^{2}$, Tomoji Mashimo ${ }^{1,2}$

${ }^{1}$ IEXAS, Grad Sch of Med, Osaka Univ. ${ }^{2}$ Genome editing R\&D center, Osaka Univ.

CRISPR/Cas9, the valuable genome editing technology, has made it possible to genetically modify a wide range of experimental animals such as drosophila, zebrafish, mouse, and rat. We have already established CRISPR-based technologies to produce genome edited rats with knockout and knock-in alleles. Especially, the preparation of large cassette knock-in rats using zygote electroporation(EP) is the advantage of our facility.

NBRP-Rat as a part of the National BioResource Project in Japan has started since 2002. The main roles of the NBRP-Rat are the collection, preservation, and supply of so many various rat strains. In 2017, Osaka University has joined this project as the central facility for preserving and distributing immunodeficient SCID rats.

We also the member of Platform of advanced Animal Model Support established by MEXT in Japan. This platform aims to support researchers who want to generate and to analyze various gene edited animals. In 2016, Institute of experimental animal sciences and Mashimo Lab. of Osaka University have participated in this platform as the largest center for gene-modified rats.

Here we report efficient and simple protocols to produce large cassette knock-in or conditional knockout rats with EP. These methods enable us to achieve the project's goal and to expand the potential of rat models. 


\title{
0-57 New Japan Mouse Clinic in RIKEN Bioresource Research Center
}

\author{
Masaru Tamura \\ Japan Mouse Clinic, Technology and Development Team for Mouse Phenotype Analysis, RIKEN \\ Bioresource Research Center, Japan
}

The Japan Mouse Clinic (JMC) has launched in 2008, and we conducted the early-onset comprehensive phenotype pipeline in RIKEN BRC. It has been built in with procedures based on IMPReSS (International Mouse Phenotyping Resource of Standardized Screens). We also participate to the International Mouse Phenotyping Consortium (IMPC, www. mousephenotype.org), which is an international scientific endeavor to create and characterize the phenotype of 20,000 knockout mouse strains. Recently, we focused on the late-onset phenotypes in mouse strains, and are progressing to construct the aging phenotyping pipeline to analyze the knockout mouse phenotype by agerelated manner. From this April, our team will be a new organization, and has plans to provide a new phenotyping pipeline. In this presentation, we would like to report this new pipeline in addition to the early-onset comprehensive and aging phenotype pipelines. We also would like to report on attempts to provide international standards phenotypic analysis to scientific communities.

\section{0-58 Behavior analysis of ASD model mice using comprehensive and multidimensional behavioral test battery}

\author{
Ikuko Yamada, Tomoko Kushida, Tomohiro Suzuki, Kimio Kobayashi, Ikuo Miura, \\ Tamio Furuse, Shigeharu Wakana
}

BioResource Center, RIKEN, Tsukuba, Japan

Recently, there has been an increase in patients with developmental disorders, which have become a social problem. There are various hypotheses regarding the etiology of these disorders, however, the concrete onset mechanisms are unclear. We have established a multidimensional behavioral phenotyping pipeline for the developmental disorder model, such as Autism Spectrum Disorder (ASD), in mice. In this study, we performed behavioral analyses of three strains of Grin 1 missense mutant, Shank2 KO, Nlgn3 KO.

Behavioral tests were conducted in order of following, light/dark transition test (6w), openfield test $(\mathrm{OF}: 7 \mathrm{w})$, Crawley's social interaction test (CSI: $8,9 \mathrm{w}$ ), home-cage activity test (HA: 10-11w), Y-maze test (12w), fear conditioning test $(13 \mathrm{w})$, and pre-pulse inhibition test $(14 \mathrm{w})$. As a result, hyper activity was observed in all three mutants in the OF. Whereas in the HA, hyper activity observed in the Grinl mutant and Shank2 KO was not in Nlgn3 KO. Also, in CSI, sociality was decreased in Grin 1 mutant and Shank2 KO, but not in Nlgn3 KO. Furthermore, in Y-maze test, alternation rate was decreased only in Nlgn3 KO.

These results indicate that multidimensional phenotype was evaluated by using our pipeline. Various model mice will be developed by analyzing more mutants using this test battery. It is expected that the results obtained from these will help to elucidate the etiology of mental disorders and developmental disorders. 


\title{
0-59 \\ Myosin VI haploinsufficiency reduced the hearing abilities in mice
}

\author{
Yuta Seki ${ }^{1}$, Hiroshi Shitara ${ }^{1}$, Yuki Miyasaka ${ }^{1,2}$, Sari Suzuki ${ }^{1,3}$, Shumpei Yasuda ${ }^{1}$, \\ Yoshiaki Kikkawa ${ }^{1}$ \\ ${ }^{1}$ Tokyo Metropolitan Institute of Medical Science, Tokyo, Japan ${ }^{2}$ Nagoya university, Nagoya, Japan \\ ${ }^{3}$ Fukuoka university, Fukuoka, Japan
}

In humans, heterozygous and homozygous null mutations of MYO6 cause postlingual progressive and prelingual profound hearing loss, respectively. We produced novel Myo6 null mutant (Myo6 $\left.{ }^{\text {p.Thrl3fs }}\right)$ mice using genome editing and analyzed the hearing phonotypes of homozygous and heterozygous mutants. Homozygous Myo6null mutants of mice also develop congenital profound hearing loss, which is caused by the fusion of stereocilia of both inner and outer hair cells. We confirmed that heterozygotes developed progressive hearing loss at high frequency sounds by measurements of the auditory brainstem response (ABR). Moreover, amplitudes of the ABR peaks in sound stimuli at all frequencies were clearly reduced in heterozygotes compared with wildtype mice. In addition, the distortion product otoacoustic emission levels were also reduced in heterozygotes. Next, we investigated the morphological phenotypes in heterozygotes. Although abnormalities were not detected in stereocilia, we found that ribbon synapses of the inner hair cells from the base area of cochlea were significantly reduced in heterozygotes. Moreover, the loss of outer hair cells in heterozygotes occurred at an earlier age compared with wild-type mice. Thus, our study suggested that MYO6 haploinsufficiency affects the detection of sounds in mice as well as humans.

\section{0-60 Development of Imaging Technology for fibrosis-visualizing model mice}

\author{
Yoshihiro Miwa ${ }^{1,2}$, Junko Tanaka ${ }^{1}$, Tomoki Sakasai ${ }^{3}$, Seiya Mizuno ${ }^{1,2}$, \\ Masafumi Muratani ${ }^{1}$, Fumihiro Sugiyama ${ }^{1,2}$, Satoru Takahashi, ${ }^{1,2}$ \\ ${ }^{1}$ Faculty of Medicine, University of Tsukuba, Tsukuba, Japan ${ }^{2}$ LARC, University of Tsukuba, Tsukuba, \\ Japan ${ }^{3}$ Graduate School of Comprehensive Human Sciences, University of Tsukuba
}

Tissue fibrosis arises related to injury by various diseases such as chronic inflammation, virus infection, diabetes and so on. Progress of fibrosis decreases functions of organs and lowers the QOL of patients. There is no effective treatment for fibrosis and recently two kinds of new preventive medicines have been developed and approved but further efforts for drug discovery are required. Establishment of model mice in which the onset of fibrosis can be visualized non-invasively is promising to enhance fibrosis research. NIR light with wavelength from 650 $\mathrm{nm}$ to $900 \mathrm{~nm}$ is called optical window and suitable for mammalian non-invasive imaging. Therefore, we have established new mice expressing NIR fluorescent protein, iRFP-based degradation-controllable probe, TetDeg-iRFP driven by collagen promoter. In parallel, we have succeeded to develop the novel technology enabling live imaging of collagen fiber formation. We will report these new methods and discuss the usefulness of them. 


\title{
0-61 Identification of downstream genes of the uterine SOX17 around the receptive period
}

\author{
$\bigcirc$ Yoshikazu Hirate ${ }^{1}$, Kana Hayakawa ${ }^{1}$, Yuki Nakano ${ }^{1}$, Mami Uemura ${ }^{1,2}$, Kento Miura² \\ Yoshiakira Kanai $^{2}$, Masami Kanai-Azuma ${ }^{1}$ \\ ${ }^{1}$ Cent for Exp Anim, Tokyo Medical and Dental University, Tokyo, Japan ${ }^{2}$ Dept of Vet Anat, The \\ University of Tokyo, Tokyo, Japan
}

Implantation is a complicated phenomenon with drastic morphogenetic and signaling changes. We have revealed that Sox 17 heterozygous mutant female mice are subfertile due to impaired implantation. It has also been reported that human SOX17 is involved in successful implantation. However, the role of SOX17 in implantation remains largely unknown. Here, we report dynamic expression of Sox 17 and identification of candidate genes downstream of SOX17 in the receptive uterus. Uterine Sox 17 showed the highest expression in the luminal epithelium at the receptive period. Microarray analysis identified 55 genes, whose expressions increased to more than 2-fold at the receptive period compared to those at day 1 and decreased to less than half in Sox 17 heterozygous mutant. Gene ontology analysis revealed that these genes are mostly related to the extracellular functions. Of these, Areg a gene encoding an EGFR ligand showed the most drastic change. Areg showed 304-fold induction in the receptive period, whereas its expression was undetectable in Sox 17 heterozygous mutant. Immunological staining revealed implantation-site specific expression of AREG in normal pregnancy. Given that EGFR is expressed in the blastocyst, it is suggested that SOX17 upregulates Areg at the implantation site and leads to adhesion of the blastocyst to the uterus.

\section{0-62 A missense mutation of Mip induces microphthalmia in rats}

\author{
Shumpei Yasuda ${ }^{1}$, Kei Watanabe ${ }^{1}$, Kenta Wada ${ }^{2}$, Takashi Kuramoto ${ }^{3}$, \\ Tomoji Mashimo $^{4}$, Yoshiaki Kikkawa ${ }^{1}$ \\ ${ }^{1}$ Tokyo Metropolitan Institute of Medical Science, Tokyo, Japan ${ }^{2}$ Tokyo University of Agriculture, \\ Abashiri, Japan ${ }^{3}$ Kyoto University, Kyoto, Jaspan ${ }^{4}$ Osaka University, Osaka, Japaqn
}

MIP plays a role in water channel activity in the plasma membrane of lens fiber cells. Previous studies have reported that the mutations in $M I P / M i p$ are responsible for cataract in humans, mice and rats. Here, we report that Mip GI7IR/GI7IR homozygous mutants also develop microphthalmia in rats. We measured sizes of eye and lens in Mip ${ }^{\text {GI7IR/GI7IR }}$ homozygotes and compared the sizes with wild-type, Mip ${ }^{G 171 R /+}$ heterozygotes, and Mip G171R/- compound heterozygotes with MIP null mutant. The sizes

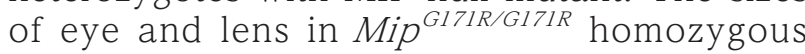
rats become atrophied to $85 \%$ and $68 \%$, respectively, compared with wild-type rats. In addition, the sizes of lens in Mip ${ }^{\text {G171R/- }}$ compound heterozygotes were $94 \%$ of those of wild-type rats. Next, we detected the Mip transcript levels in eye. The relative amount of Mip transcript in the eyes of $\mathrm{Mip}^{\text {GI7IR/+}}$, Mip ${ }^{\text {GI7IR/GI7IR }}$, and Mip ${ }^{\text {GI7IR/- }}$ rats were approximately $62 \%, 36 \%$, and $24 \%$ of the levels of wild-type, respectively. Meanwhile, the expression of MIP protein was detected in lens fiber cells as well as that of wild-type rats. By construction of a mutant model, we simulated the probability for the MIP ${ }^{\text {G17lR }}$ substitution within $\mathrm{H} 5$ transmembrane domain to charge and crash into the amino acid residue of $\mathrm{H} 4$ domain. Therefore, we speculated that structural change of MIP protein by MIP ${ }^{\mathrm{G} 171 \mathrm{R}}$ mutation causes of microphthalmia in rats. 


\title{
0-63 Induction of seizure and EEG recording in Hen1-knockout rats.
}

\author{
Ai Nishitani ${ }^{1}$, Kunisawa Naofumi ${ }^{2}$, Sato Kazuaki ${ }^{3}$, Yoshida Yusaku ${ }^{4}$, Suzuki Toshiro ${ }^{4}$, \\ Sakuma Tetsushi ${ }^{5}$, Yamamoto Takashi ${ }^{5}$, Asano Masahide ${ }^{1}$, Ohno Yukihiro ${ }^{2}$, Kuramoto Takashi ${ }^{1}$ \\ ${ }^{1}$ Institute of Laboratory Animals, Graduate school of Medicine, Kyoto University ${ }^{2}$ Laboratory of Pharmacology, Osaka University of \\ Pharmaceutical Sciences ${ }^{3}$ Department of Neurology, Graduate School of Medicine, Kyoto University ${ }^{4}$ Research Department for Experimental \\ Animals, Biotechnical Center Japan SLC, Inc. ${ }^{5}$ Department of Mathematical and Life Science, Graduate School of science, Hiroshima University
}

HCN1 channel is activated by hyperpolarization and permeates cations. Here, we induced seizure and recorded EEG in $H c n 1$-knockout rats ( $H c n 1$ $\mathrm{KO}$ rat).In febrile seizure, no difference was seen between Hcn 1-KO and F344/NSlc rats. In Pentylenetetrazol (PTZ) induced seizure, lethal dose in Hcn 1-KO was lower than that of F344/Nslc rats. This result indicated that seizure susceptibility to PTZ increased in Hcn 1$\mathrm{KO}$ rats. In EEG recording, the spike-wave discharges (SWDs) were found in Hcnl-KO rats.
During the SWD, impairment of consciousness and shaking of the jaw were seen. The SWDs were suppressed by ethosuximide (suppressing absence seizure) but not phenytoin (suppressing tonic convulsion). These findings indicated that Hcn 1-KO rats exhibited absence-like seizure. In conclusion, Hcnl is involved in seizure and epilepsy. Hcn $1-\mathrm{KO}$ rats can be used to understand mechanism underlying seizure and epilepsy.

\section{O-64 Effects of salt intake on blood pressure and urinary isoprostane in Wistar Kyoto rats}

\author{
$\bigcirc$ Kohei Kawakami $^{1}$, Hiroyuki Matsuo ${ }^{1}$, Kazuo Yamada ${ }^{2}$, Haruo Takeshita $^{2}$, \\ Takaya Yamada ${ }^{1}$ \\ ${ }^{1}$ Department of Experimental Animals, Shimane University, Shimane, Japan ${ }^{2}$ Department of Legal \\ Medicine, Shimane University, Shimane, Japan
}

The characteristic of salt-sensitive hypertension is blood pressure elevation corresponding with salt intake, and brain, heart, and kidney impairment readily develops. Although spontaneously hypertensive rats (SHR) were reported to be saltsensitive, the control Wistar Kyoto rats (WKY) have not been investigated in detail. In this study, we investigated the effects of salt intake on blood pressure and urinary isoprostane in WKY. Eight-week-old male WKY were fed a normal- (0.5\% NaCl, control), medium- (4\% $\mathrm{NaCl})$, or high- $(8 \% \mathrm{NaCl})$ salt diet for 12 weeks. Regarding test items, the body weight and blood pressure were measured every other week. In order to measure urinary isoprostane levels, urine was collected at 4, 8, and 12 weeks. Weight gain started to decrease at 2 weeks in the $8 \% \mathrm{NaCl}$ group, and a significant difference was noted between it and the control group. The blood pressure elevation effects became marked in the $8 \% \mathrm{NaCl}$ diet group at 6 weeks after administration, demonstrating a significant difference. In addition, urinary isoprostane levels were significantly higher in the $8 \% \mathrm{NaCl}$ group at 4, 8, and 12 weeks. High-salt diet loading tended to cause high blood pressure in a concentrationdependent manner. The results suggest that the increase in blood pressure during dietary saltinduced hypertension continues longer with $8 \%$ $\mathrm{NaCl}$ than with $4 \% \mathrm{NaCl}$ in normotensive WKY. 


\title{
0-65 Establishment of novel murine pleomorphic rhabdomyosarcoma cell lines with KRasG12V;p53\%.
}

\author{
$\bigcirc$ Hiromitsu Saito, Noboru Suzuki \\ Dept. Animal Functional Genomics of Advan. Sci. Res. Center, Org. for Prom. of Regional Innovation, \\ Univ. of Mie, Japan
}

Pleomorphic rhabdomyosarcomas (pRMSs) arise predominantly in the skeletal musculature of adults, and are typically associated with poor prognosis; thus, it is critical to develop experimental systems to understand the cellular and molecular mechanisms that give rise to pRMSs. In this study we established murine tumorigenic cell lines, designated RMS3, RMS310 and RMSg2. RMS3 was generated from a primary culture of a p53-deficient KRrasG12V tumor induced in the femur. RMS310 was established as an RMS3 derivative by limiting dilution of tumor cells from a lung metastatic colony of RMS3. RMSg2 was an RMS310 derivative established by limiting dilution of tumor cells from a lung metastatic colony of GFP-expressing RMS310. All cell lines exhibited stable tumorigenicity and gave rise to pRMSs characterized by bizarre giant cells, positive for desmin by subcutaneous inoculation. The RMSg2 cell line is highly metastatic and were able to colonize the lung from tumorigenic cells injected in the orbital vein and subcutaneously. RT-PCR revealed that all cell lines displayed gene expression profiles similar to fibro-adipogenic progenitors (FAPs) markers Sca-1, PDGFR $\alpha$, Adam12, and Tcf4, but negative for SC markers Pax7 and Myf5. This suggest that these p53 deficient KRasG12V tumor cell lines have FAPs like features, but not SCs.

\section{0-66} Effect of Seaweed-Derived $\beta$-Glucan against the progression of gastric cancer precursor lesion

$\bigcirc$ Mark Joseph M. Desamero ${ }^{1,2}$, Shigeru Kakuta1 ${ }^{1}$ James K. Chambers³, Kazuyuki Uchida ${ }^{3}$, Jun Nakayama ${ }^{4}$, Satoshi Hachimura ${ }^{5}$, Hiroyuki Nakayama ${ }^{3}$, Shigeru Kyuwa ${ }^{1}$

${ }^{1}$ Dept. Biomed Sci.,. Grad. Sch. Agr. Life Sci., Univ. Tokyo, Tokyo, Japan ${ }^{2}$ Dept. Basic Vet. Sci., Col. Vet. Med., Univ. Philippines Los Banos, Laguna, Philippines ${ }^{3}$ Dept. Vet. Pathol. Grad. Sch. Agr. Life Sci., Univ. Tokyo, Tokyo, Japan ${ }^{4}$ Dept. Mol. Pathol., Shinshu Univ. Grad. Sch. Med., Matsumoto, Japan ${ }^{5}$ Res. Cntr. Food Safety., Grad. Sch. Agr. Life Sci., Univ. Tokyo, Tokyo, Japan

$\beta$-glucan refers to a heterogeneous group of storage polysaccharides containing $\beta$ -1,3-D-linked glucose polymers with branches connected by either $\beta-1,4$ or 1,6 glycosidic linkage, and has multifunctional biological activities. In this study, we have employed a unique animal model of early gastric dysplasia, A4gnt $\mathrm{KO}$ mice to investigate the putative gastro-protective activity of brown seaweedderived $\beta$-glucan (Laminaran) against the progressive development of precancerous lesions. Administration of $100 \mathrm{mg} / \mathrm{kg}$ BW Laminaran resulted in a significant amelioration of the gross gastric mucosal swelling and PMNLs infiltration, and substantially reduced proliferative and angiogenic processes. Furthermore, Laminaran treatment considerably reversed the A4gnt $\mathrm{KO}$-induced alteration in the gene expression profile of selected cytokines. Collectively, our present findings indicate that $\beta$-glucan can potentially restrain the progressive development of precancerous lesions to mediate their tissuepreserving activity. 


\title{
0-67 Functional analysis of the genetic polymorphism in $p 19^{A r f}$
}

\author{
Megumi Saito ${ }^{1,2}$, Kazuhiro Okumura ${ }^{1}$, Eriko Isogai ${ }^{1}$, Yuichi Wakabayashi ${ }^{1}$ \\ ${ }^{1}$ Department of Carcinogenesis Research, Division of Experimental Animal Research, Chiba Cancer Center Research Institute, Chiba, Japan \\ ${ }^{2}$ Department of Molecular Biology and Oncology, Graduate School of Medical and Pharmaceutical Sciences, Chiba University, Chiba, Japan
}

In recent study, we successfully mapped highly significant QTLs for late stage papillomas $(>6 \mathrm{~mm}$ ) and identified Stmm3 (Skin tumor modifier of MSM 3) locus on Chromosome 4 by crossing a resistant Japanese wild-derived inbred strain MSM/Ms with a susceptible strain $\mathrm{FVB} / \mathrm{N}$ and subjected to the two-stage skin carcinogenesis protocol using DMBA/TPA. And we mapped Stmm3 locus to $88-93 \mathrm{Mb}(5 \mathrm{Mb})$ on Chromosome 4. This locus on Chromosome 4, which maps near the Cdkn2a/p19 ${ }^{\text {Arf }}$ gene, was genetically $p 53$-dependent, and was not seen in $p 53^{+/}$backcross mice. There are nonsynonymous polymorphisms in $p 19^{\text {Arf }}$ between
FVB/N and MSM/Ms mice. Therefore, we generated cell lines overexpressing $\mathrm{p} 19^{\text {Arf MSM }}$ or p19 $9^{\text {Arf FVB }}$, and biological assays were performed using these cell lines. As a result, p19 $9^{\text {Arf MSM }}$ was more effective in inducing $G_{1}$ arrest and protein stability was higher than p19 $9^{\text {Arf FVB }}$ by TPA treatment. Furthermore, we generated $p 19^{\text {Arft } 1-}$ $(\mathrm{FVB} / \mathrm{N} \times \mathrm{MSM} / \mathrm{Ms}) \mathrm{Fl}$ mice to investigate the function of $p 19^{\text {Arf }}$ in in vivo. Skin carcinogenesis experiments showed that $p 19^{\text {Arf } \mathrm{MSM}}$ allele confers resistance to larger papillomas. These data suggested that the genetic polymorphism in p19 ${ }^{\text {Aff }}$ might be expected to be a controlling the transition to large papillomas in MSM/Ms.

\section{0-68 Simplified enriched environment suppresses cancer progression in mice}

\author{
$\bigcirc$ Jun Watanabe ${ }^{1,2}$, Hideto Matsuhashi², Nobuyuki Kagami², Satoru Arata ${ }^{1,2}$ \\ ${ }^{1}$ Center for biotechnology, Showa University, Tokyo, Japan ${ }^{2}$ Center for Laboratory Animal Science, \\ Showa University, Tokyo, Japan
}

Recently, mice housed in an enriched environment (EE) are reported to show inhibition of tumor development. However, the mechanisms underlying increased cancer resistance in the EE are still unclear, which may be due to poor reproducibility of the results because of complicated EE structure. Here, we examined the effects of a simplified EE model consisting only of a nesting shelter and a running wheel on cancer development in mice. The results indicated that, similar to the complex EE, the simplified EE promoted motor function and alleviated anxiety in mice. On the other hand, simplified EE did not change the cognitive function. Furthermore, simplified EE inhibited tumor growth and metastasis, which corresponded to the increase in average body temperature, especially at the light-to-dark switching, compared to control. Interestingly, mice in the simplified EE had decreased brown adipose tissue (BAT) mass and significant upregulation of the mRNA expression of the Ucpl and Adr3b genes coding for uncoupling protein 1 and $\beta$-adrenergic receptor, respectively, in BAT at the light-to-dark switching. These data suggest that the anti-tumor effects of eustress modeled by the simplified EE are due to modulation of thermogenesis. Our findings indicate that the established simplified EE model could be a useful tool for studying anti-tumor effects of enriched environment. 


\title{
0-69 Wapl develop oncogenic activity with HPV E6/E7 in mice
}

\author{
$\bigcirc$ Katsuyoshi Kumagai ${ }^{1}$, Masakatsu Takanashi ${ }^{2}$, Shin-ichiro Ohno ${ }^{2}$, Katsuko Sudo ${ }^{1}$, \\ Masahiko Kuroda ${ }^{2}$ \\ ${ }^{1}$ University-related Facilities Pre-clinical Research Center, Tokyo Medical University, Tokyo, Japan \\ ${ }^{2}$ Department of Pathology, Tokyo Medical University, Tokyo, Japan
}

Human papillomavirus (HPV) is the primary causal agents responsible for the development of cervical cancer. Invasive carcinomas develop after only a small portion of precursor lesions infected with HPV. Therefore, additional genetic factors after HPV infection are thought to play an important role in the initiation and progression of cervical carcinoma. Wapl deficiency causes chromatin compaction by stabilizing cohesin on DNA which it is an essential regulator of chromatin structure and chromosomal stability. Moreover, High-level expression of hWAPL (the human homologue of wapl) was observed in cervical cancers; it is necessary to reveal about the regulation of development of cervical cancer by E6/E7 and Wapl. In this study, we have demonstrated that Wapl has the characteristics of an oncogene and is associated with uterine cervical cancer. First, we have demonstrated that transduction of Wapl with E6/E7 is sufficient for the development of canceration in epithelium cells. Here we show that transduction of Wapl with E6/E7 expression causes increase not an only number of colonies but also the formation of colonies. Furthermore, Ki-67 and p16 increased in Wapl and E6/E7 expressing mice by immunostaining analysis. These results suggest that Wapl could be developed not only oncogenic activity in epithelium cells with E6/E7 but also canceration of female mice.

\section{0-70 Evaluation of Common marmoset as an animal model for Zika virus infection}

Satoshi Taniguchi ${ }^{1}$, Meng Ling Moi ${ }^{2}$, Nor Azila Muhammad Azami ${ }^{1}$, Yasushi Ami ${ }^{3}$, Yuriko Suzaki ${ }^{3}$, Noriyo Nagata ${ }^{4}$, Naoko Iwata ${ }^{4}$, Tomohiko Takasaki ${ }^{5}$, Shigeru Tajima ${ }^{1}$, Chang-Kweng Lim¹․ Masayuki Saijo ${ }^{1}$ ${ }^{1}$ Department of Virology I, National Institute of Infectious Diseases, Tokyo, Japan ${ }^{2}$ Institute of Tropical Medicine, Nagasaki University, Nagasaki, Japan ${ }^{3}$ Division of Experimental Animal Research, National Institute of Infectious Diseases,Tokyo,Japan ${ }^{4}$ Department of Pathology, National Institute of Infectious Diseases,Tokyo,Japan ${ }^{5}$ Kanagawa Prefectural Institute of Public Health, Kanagawa, Japan

Zika virus (ZIKV) belongs to the family Flavividae, genus Flavivirus. Previous studies reported that infection by ZIKV during pregnancy can cause congenital ZIKV infection to their infants. In this study, we aimed to evaluate common marmoset as an animal model for ZIKV infection. Six marmosets were subcutaneously inoculated with ZIKV MR766-NIID strain or PRVABC59 strain. Blood, oral swab, and urine samples were collected at day 0,2, 4, 8, 10, and 14 post-infection and viral genome loads in these samples were determined using the quantitative RT PCR assay. The viral genome loads in the PRVABC59 infected marmosets were higher than those of MR766-NIID infected marmosets. At day 14 post-infection, viral genomes were detected in urine samples but not in serum samples. These results were consistent with previous studies on human ZIKV infection. Our results demonstrated that common marmosets can be a reliable nonhuman primate animal model for ZIKV infection. 


\title{
0-71 Rat hepatitis E virus infection during pregnancy
}

\author{
Satoshi Kunita ${ }^{1}$, Masaharu Takahashi ${ }^{2}$, Hiroaki Okamoto ${ }^{2}$ \\ ${ }^{1}$ Center for Experimental Medicine, Jichi Medical University, Tochigi, Japan ${ }^{2}$ Division of Virology, \\ Department of Infection and Immunity, Jichi Medical University, Tochigi, Japan
}

We have previously reported that rats infected with wild rat-derived hepatitis E virus (rat HEV) are useful models of HEV infection. In this study, we analyzed virus load in pregnant rats and vertical transmission of rat HEV to reproduce severe cases of HEV infection in pregnant women.

METHODS: $2 \times 10^{6}$ copies/rat of rat HEV (strain rat ELOMB-131L) were intravenously inoculated into pregnant F344 rats at the 5 days or 12 days post-mating and non-pregnant rats. Sera, feceses and livers from the infected rats and infants were used for measuring anti-rat HEV IgG and IgM by ELISA and rat HEV RNA by real-time RT-PCR. RESULTS: Rat HEV-infected pregnant rats gave birth normally without showing abnormal clinical signs. In the rats infected during late pregnancy, duration of viral excretion into feces was extended compared to those in rats infected in early pregnancy and non-pregnant controls. Transient elevation of serum AST was observed in rats infected during late pregnancy. Vertical transmission of rat HEV was not detected.

DISCUSSION: In rat HEV-infected pregnant rat model, prolonged viral proliferation and transient liver injury were observed without severe hepatitis. Because the pregnancy period of rats is as short as about 21 days, it is obstacle that virus proliferation and the influence of antibodies against viruses cannot be observed within such a short period of time during pregnancy in rat model.

\section{0-72 Studies on Transmission of Mouse Hepatitis Virus Strains in Laboratory Mice}

\author{
Yuki Sugiura, Shigeru Kakuta, Shigeru Kyuwa \\ Department of Veterinary Medical Science, the University of Tokyo, Tokyo, Japan
}

Mouse hepatitis virus (MHV) is a major concern in laboratory animal facilities. It is useful to understand the manner of MHV transmission to prevent animals from MHV infection. There is a difference in the transmissibility among MHV strains namely; MHV-JHM, MHV-NuU and MHV-Y with the last being the most contagious strain. In the present study, the factors involved in the strain-specific difference in the transmissibility of MHV were examined. We inoculated intranasally mice with MHV-Y or MHV-NuU. One month later, sera were collected for ELISA test to compare the seroconversion rate between MHV strains. Fresh feces were collected every other day for real time RT-qPCR analysis. We also inoculated mice with fecal suspension from MHV-Y or MHV-NuU infected mice collected at every other day. Sera and fresh feces were collected same as above. The lowest viral load of MHV-NuU required for seroconversion was significantly lower than that of MHV-Y. The amount of MHV-Y RNA in the feces was, at most approximately 40 fold higher than that of MHV-NuU. The groups of mice inoculated with the suspension of feces from MHV-Y infected mice showed seroconversion and no groups of MHV-NuU showed seroconversion. Although the amount of virus excrete in the feces is one of the most important factors that determine the degree of difference in the transmissibility among MHV strains which are largely transmitted via the fecal-oronasal route, we cannot exclude the possibility that other factors. 


\title{
0-73 A survey of Rat polyomavirus2 in laboratory rats in Japan
}

\author{
Nobuhito Hayashimoto ${ }^{1}$, Masahiko Yasuda ${ }^{2}$, Ritsuki Uchida ${ }^{3}$, Miho Yamamoto $^{4}$, \\ Masafumi Yamamoto', Kenji Kawai ${ }^{2}$ \\ ${ }^{1}$ ICLAS Monitoring Center, Central Institute for Experimental Animals, Kawasaki, Japan ${ }^{2}$ Pathological \\ Analysis Center, CIEA, Kawasaki, Japan ${ }^{3} \mathrm{JAC}$ Inc. Tokyo, Japan ${ }^{4}$ WDB Inc. Tokyo,Japan
}

Rat polyomavirus2 (RatPyV2) is known as a causative agent of severe respiratory infection in immunedeficient rats like as X-linked severe combined immunedeficiency (X-SCID) rats. Recently, population survey found antibodies to RatPyV2 in $32 \%$ of immunocompetent rats used in biomedical research in U.S.A. The actual figure of prevalence of RatPyV2 in laboratory rats in Japan is unknown. In this study, we surveyed RatPyV2 in immunocompetent rats in experimental facilities in Japan. This survey was conducted in 746 rats derived from 271 facilities in a total in Japan. Harderian glands and salivary glands are collected from autopsied rats and used as samples for PCR. These organs derived from RatPyV2 positive rats were also examined histopathologically. As a result, 35 rats derived from 32 facilities were positive for RatPyV2. The contamination rates were $11.8 \%$ (number of facilities) and 4.6\% (number of animals). Twenty-five rats were positive for RatPyV2 only from salivary glands, and 5 rats were positive for RatPyV2 only from Harderian glands. And 5 rats were positive for RatPyV2 from both of them. No histopathological changes were observed for all samples derived from RatPyV2 positive rats. These results suggest a potential of RatPyV2 positive immunocompetent rats as a contamination source for immunedeficient rat colony.

\section{0-74 Examination to change chromogenic substrate solution of MONILISA $^{\circledR}$}

\author{
Mai Tanaka ${ }^{1}$, Tomoko Ishida ${ }^{1}$, Miyuki Ozawa ${ }^{2}$, Asami Kin², Takayasu Noguchi ${ }^{2}$, \\ Nobuhito Hayashimoto ${ }^{1}$ \\ ${ }^{1}$ Central Institute for Experimental Animals, Kawasaki, Japan ${ }^{2}$ Wakamoto Pharmaceutical CO., Ltd.
}

MONILISA ${ }^{\circledR}$ is the ELISA (the enzyme-linked immunosorbent assay) kit assaying antibodies which Central Institute for Experimental Animals and Wakamoto Pharmaceutical Co., Ltd.. developed together. Chromogenic substrate solution of MONILISA ${ }^{\circledR}$ is used o-Phenylenediamine (OPD). OPD is categorized deleterious substances and its require preparation before use. One the other hands, 3,3',5,5'-tetramethylbenzidine (TMB) is low hazard and no require preparation before use. In this study, we examined whether TMB will be substitute for OPD.

The test items are Sendai virus, Mouse Hepatitis virus, Myccoplasma pulmonis, Clostridium piliforme and Hanta virus. Antibody test by
ELISA method was carried out using the antigen plate of MONILISA ${ }^{\circledR}$. We used positive control, antiserum diluted 40 to 5,120 times and 24 negative serum for samples.

OD (optical density) using positive control of 5 test items were 0.369 to 1.061 with OPD and 0.728 to 2.281 with TMB. TMB indicated higher OD than OPD. Therefore, the reaction volume was reduced to $1 / 2$ of $\operatorname{OPD}(200 \mu \mathrm{l}$ to $100 \mu)$. As a result, OD of antiserum diluted 40 times were 0.900 to detection limit using OPD $(200 \mu$ l) and 0.651 to detection limit using TMB (100 $\mu$ 1). All Negative serum samples were negative. TMB showed equivalent result to OPD by haling reaction volume. Using TMB for MONILISA ${ }^{\circledR}$ can be expected for reduction of sample volume. 


\title{
0-75 Influence of drinking water on colonization dynamics of altered Schaedler flora in IQI mice
}

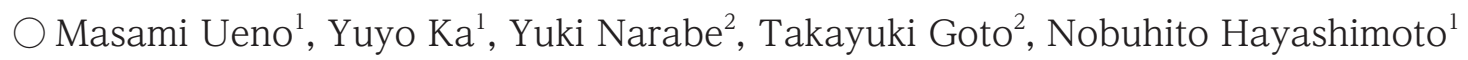 \\ ${ }^{1}$ Central Institute for Experimental Animals, Kanagawa, Japan ${ }^{2}$ Technical Service Department, CLEA \\ Japan, Inc., Shizuoka, Japan
}

The altered Schaedler flora (ASF), consisting of eight bacterial species, is common globally as minimum microbiota that is sufficient to establish normal physiological functions in the gut of mice. Recently, we introduced ASF, and started the basic study for global harmonization. In this study, we evaluated of influence of drinking water on colonization dynamics of 8 strains in gnotobiotic ASF-colonized IQI mice that drunk each of water derived from two facilities.

Eighteen male and 18 female 3-week-old IQI mice were divided into two groups and given each of water derived from two facilities (CIEA and Technical Service Dept., CLEA Japan, Inc.). The colonization change of the ASF strains caused by aging was assessed using fecal samples obtained each week from 3-12-week-old IQI mice. Stomach, small intestine, cecum and colon contents were collected from same mice at 12-week-old to analyze the distribution patterns of the ASF strains. Extracted DNA from each sample was used for real-time quantitative PCR based on SYBR Green I.

As a result, the ASF strains were detected at high concentration in feces of 3-week-old mice, and the numbers of bacteria were no rapid change up to 12-week-old. Also, there were no significant differences in colonization levels of the ASF strains between two drinking water groups. In addition, we will evaluate distribution changes of the ASF strains in gut of IQI mice.

\section{0-76 Comparison of gut microbiota between post-human fecal microbiota transplantation pigs and humans}

\author{
$\bigcirc$ Hiromasa Hara ${ }^{1}$, Manabu Nagayama ${ }^{2,3,4}$, Wataru Suda ${ }^{3}$, Hiroaki Shibata ${ }^{1,5}$, Takahiro Ohnuki ${ }^{5}$, Shuji Hishikawa ${ }^{5}$, \\ Satoshi Kunita ${ }^{5}$, Tomoyuki Abe ${ }^{1,5}$, Hideki Uosaki ${ }^{1,5}$, Koji Atarashi ${ }^{3}$, Kenya Honda ${ }^{3,4}$, Yutaka Hanazono ${ }^{1,5}$ \\ ${ }^{1}$ Division of Regenerative Medicine, Jichi Medical University ${ }^{2}$ Division of Gastroenterology, Jichi Medical University ${ }^{3}$ Keio \\ University School of Medicine ${ }^{4}$ RIKEN Center for Integrative Medical Sciences ${ }^{5}$ CDAMTec, Jichi Medical University
}

Pigs have a gastrointestinal tract and digestive physiology similar to those of humans compared to mice. Thus, post-human fecal microbiota transplantation (post-hFMT) pigs would more faithfully recapitulate the gut microbiota in the human body. Here, we performed hFMT to pigs and compared the gut microbiota among posthFMT pigs, humans and SPF pigs. Piglets were delivered and reared in germfree conditions, and human feces $(2.4 \mathrm{~g} / \mathrm{kg}$, po) were transplanted at day 28 of age. A variety of bacteria were detected by $16 \mathrm{~S}$ rRNA sequencing of pig fecal samples. This revealed that bacteria derived from human gut successfully colonized in the posthFMT pigs. Gut microbiota of the post-hFMT pigs became more similar to that of humans compared to that of SPF pigs, but was still far different from that of humans. It is known that diets affect gut microbiota. Therefore, we fed the post-hFMT pigs with human diets to see whether the gut microbiota became more similar to that of humans. The gut microbiota after human diets, however, remained different from that of humans. These results indicated that there are species specificities regarding gut microbiota regardless of diets they take. 


\title{
Characterization of [Pasteurella pneumotropica] genomic information
}

\author{
$\bigcirc$ Fumio Ike ${ }^{1}$, Ayako Kajita ${ }^{1}$, Toshiaki Nakashiba ${ }^{1}$, Yuichi Obata ${ }^{1}$, Hiraku Sasaki ${ }^{2}$, \\ Atsushi Yoahiki ${ }^{1}$
}

${ }^{1}$ RIKEN Bioresource Research Center, Tsukuba, Japan ${ }^{2}$ Juntendo University, Chiba, Japan

[Pasteurella pneumotropica] is one of spontaneous bacterial pathogens in rodents. Recently two biotypes (Jawetz and Heyl) of this bacteria were scientifically named as Rodentibacter pneumotropicus and $R$. heylii, respectively. Genomic sequencing of both species was already carried out by a few groups. Here, we reclassified genomic sequence information of both species and tried to characterize these species. Methods Genome data of Rodentibacter family species (4 data of $R$. pneumotropicus and 7 data of $R$. heylii) were obtained from NCBI to create database for local BLAST analysis. Type strain data of both species were applied to MiGap annotation pipeline. Then genome data were searched for the annotated gene names and coding regions.
Results and Discussion Our findings are, 1) both species has at least 6 rRNA operons with wobbles in each sequences. 2) Variations of nucleic acid sequences among strains are observed in $R$. pneumotropicus genomes, which mean that selecting appropriate genes may make possible to identify isolated strains. However genome of $R$. heylii is rather stable and variations of nucleic acid sequences among strains are moderate. 3) Variation of nucleic acid sequences between strains isolated from different animals is noted. Consequently molecular diagnosis should be addressed by careful consideration of copy number of the relevant gene in the genome and the genetic wobble.

\section{0-78 Fecal transplantation for the treatment of Clostridium difficile infection in common marmosets}

\author{
$\bigcirc$ Ryoko Nozu ${ }^{1}$, Takashi Inoue ${ }^{2}$, Kenya Sato ${ }^{2}$, Nobuhito Hayashimoto ${ }^{1}$ \\ ${ }^{1}$ Central Institute for Experimental Animals, Kawasaki, Japan ${ }^{2}$ Central Institute for Experimental \\ Animals, Kawasaki, Japan
}

Enteritis and diarrhea caused by Clostridium difficile (CD) have occasionally been observed in common marmosets. Recently, the therapeutic effects of fecal microbiota transplantation (FMT) in human patients and a marmoset have been reported. In the present study, we assessed the therapeutic effects of FMT against CD infection (CDI) in marmosets.

Frozen marmoset feces were administered to germ-free mice for safety evaluation; these mice were defined as marmoset microbiota-associated (MMA) mice. The FMT material was a mixture of cecum and feces from MMA mice; marmoset feces administered to the germ-free mice were also used. Three marmosets with recurrent CDI were used as FMT recipients. These marmosets developed diarrhea, and showed positive results in the C. diff Quik Chek complete (Alere) test more than two times. The FMT protocol was follows: antibiotic cocktail was intragastrically administered twice a day for 3 days, and a fecal suspension was also administered in a single dose, 36 hours following the final antibiotic treatment.

No curative effect was observed in marmosets with recurrent CDI following FMT using in either fecal materials from MMA mice and original marmosets. Composition analysis revealed that the fecal microbiota of recipients was not similar to the administered FMT materials. Therefore, improvements to the FMT protocol are necessary for an effective therapy against CDI in marmosets. 


\title{
0-79 Search for definitive senescence biomarkers in naturally-aged mice
}

\author{
Noboru Ogisoํㅜ Kaori Muguruma ${ }^{1}$, Satomi Takano ${ }^{1}$, Kohei Tomita ${ }^{2}$, \\ Kazumichi Yamaguchi ${ }^{2}$, Naomi Matsui ${ }^{3}$, Mitsuo Maruyama ${ }^{3}$ \\ ${ }^{1}$ Laboratory of Experimental Animals, National Center for Geriatrics and Gerontology, Obu, Japan ${ }^{2}$ KAC Corporation, \\ Kyoto, Japan ${ }^{3}$ Department of Mechanism of Aging, National Center for Geriatrics and Gerontology, Obu, Japan
}

NCGG have kept many naturally-aged animals used for gerontology and geriatric researches. If a scientist make a research using these animals, it is very important to consider the effects of various genetic and environmental factors on them. In the present study, we will report various age-related characteristics observed in our mice. Male and female C57BL/6NCrSlc mice (4-weeks-old) were purchased from Japan SLC every three months and kept over their lifetime. Physiological, behavioral and morphological analyses and blood test were performed. Body weight peaks at 18-20 months-old (M) and only male mice show rapid decrease at around 25
M. Survival rates start to decline approximately $21 \mathrm{M}$, and are somewhat lower in female aged mice. Urinary corticosterone levels are relatively higher in female mice and tend to increase with age. Rotarod performance and grip strength of aged animals show relatively low score. Enlarged seminal vesicles or splenic tumors were often found at autopsy. Blood test show that the total WBC count starts to decline at around 18 M. Various age-related changes found in our aged $\mathrm{B} 6 \mathrm{~N}$ mice can be candidate senescence indicators at individual level. We will investigate these parameters in detail and continue to search for novel biomarkers.

\section{0-80 Osteoclast differentiation and function in ASCT2-deficient mice}

\author{
$\bigcirc$ Hideki Tsumura ${ }^{1}$, Miyuki Shindo $^{1}$, Ryuji Ohkura ${ }^{2}$, Kenji Miyado $^{2}$, Xia0-Kang Li $^{3}$, \\ Masafumi Inui ${ }^{4}$, Shuji Takada ${ }^{4}$, Morihiro Ito ${ }^{5}$, Yasuhiko Ito ${ }^{5}$, Akihiro Umezawa ${ }^{2}$ \\ 'Division of Laboratory Animal Resouurces, National Research Insitute for Child Health and Develooment, Tokvo, Japan 'Department of Reproductive Biology, National Research Institute

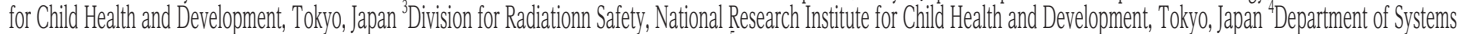 \\ BioNedicine, National Research Institute for Child Health and Development, Tokyo, Japan Department of Microbiology, College of Life and Health, Chubu University, Aichi, Japan
}

ASCT2 is a small neutral amino acid exchanger that is the most described glutamine transporter in many proliferating cells. To investigate the role of ASCT2 in osteoclast, we have established ASCT2-deficient mice by conventional genetargeting approach. Glutamine uptake was assessed in ASCT2 $2^{+/+}$and ASCT2 $2^{-/}$bone marrow cells stimulated with RANKL. The rate of glutamine uptake was reduced to $70 \%$ of glutamine uptake in ASCT $2^{+/+}$bone marrow cells. To confirm the involvement of ASCT2 in osteoclast formation, the bone marrow cells were stimulated with RANKL and mCSF, or co-culture with osteoblasts in the presence of $1,25(\mathrm{OH})^{2}$ vitamin D3 and then these cells were stained with tartrate-resistant acid phosphatase. The multinucleated osteoclast formation was severely impaired in bone marrow cells isolated from $\mathrm{ASCT}^{-/-}$mice compared with those from $\mathrm{ASCT}^{+/+}$ mice. These results suggest that ASCT2 plays an important role in the osteoclast formation. 


\title{
0-81 \\ Pathophysiological role of protein glycosylation in pancreatic islets of the ZFDM rat
}

\author{
Norihide Yokoi ${ }^{1}$, Tomohide Hayami ${ }^{1}$, Naoya Hatano ${ }^{2}$, Shihomi Hidaka ${ }^{1}$, \\ Ayako Kawabata $^{1}$, Yoshikazu Hoshino ${ }^{3}$, Masayuki Hoshino ${ }^{3}$, Susumu Seino ${ }^{1}$ \\ ${ }^{1}$ Division of Molecular and Metabolic Medicine, Kobe University Graduate School of Medicine, Kobe, Japan ${ }^{2}$ The Integrated Center for \\ Mass Spectrometry, Kobe University Graduate School of Medicine, Kobe, Japan 3Hoshino Laboratory Animals, Inc., Ibaraki, Japan
}

The Zucker fatty diabetes mellitus (ZFDM) rat strain harboring the fatty mutation (fa) in the leptin receptor gene is a novel animal model of obese type 2 diabetes. At prediabetic state (12 weeks of age), the ZFDM rats show enlarged islets, accompanied by defects in glucose metabolism and insulin secretion. Although hyperglycemia enhances protein O-GlcNAc glycosylation in islets, pathophysiological role of O-GlcNAcylation in islets of prediabetic ZFDM rats remains unknown. Here we tried to clarify role of O-GlcNAcylation in islets of prediabetic ZFDM rats. Western blotting analysis using anti-O-GlcNAc antibody revealed that O-GlcNAcylation in islets of ZFDM rats was higher than that of control lean rats. Immunoprecipitation using anti-O-GlcNAc antibody in combination with mass spectrometry using LCMS-IT-TOF identified several proteins of which O-GlcNAcylation was increased in islets of ZFDM rats. Some of them were involved in glucose metabolism and insulin production and secretion. These findings suggest that enhanced O-GlcNAcylation may cause dysregulation of glucose metabolism and insulin secretion in islets of ZFDM rats.

\section{0-82 Involvement of Nuclear Factor of Activated T-cell (NFATc2) in food allergy-inducedl OVA23-3 mice}

$\bigcirc$ Yasuko Tokunaga ${ }^{1}$, Satoki Yamada², Haruyo Adachi², Mark Joseph DESAMERO², Shigeru Kakuta ${ }^{3}$, Naoki Hayashida ${ }^{1}$

${ }^{1}$ Division of Molecular Gerontology and Anti-Aging Medicine, Department of Biochemistry and Molecular Biology, Yamaguchi University School of Medicine, Ube, Japan ${ }^{2}$ Research Center for Food Safety, Graduate School of Agricultural and Life Sciences, The University of Tokyo, Bunkyoku, Japan ${ }^{3}$ Department of Biomedical Science, Graduate School of Agricultural and Life Sciences, The University of Tokyo, Bunkyo-ku, Japan

Misdirected immune system activity induces food allergy against safe food. We discovered immune response transcription factor NFATc2 prominently suppressed the onset and progression of Huntington's disease (HD) before (Hayashida et al., EMBO J 2010; Hayashida, Exp Anim 2015). Importantly, NFATc2-deficient HD mice died of infectious diseases. In this study, we examined the roles of NFATc2 in food allergy using food allergy model OVA23-3 mice. We administrated the egg white (EW) diet to OVA23-3 and examined the NFATc2 expression in small intestine. EW diet induced food allergy and T-cell infiltration was much higher than control diet mice. NFATc2 expression was prominently increased, but it was not detected in T-cells. We also analyzed NFATc2 expression in the spleen, mesenteric lymph nodes (MLNs), and $\mathrm{CD}^{+}{ }^{\mathrm{T}}$ cells from these two tissues derived from EW diet OVA233. Compared to controls, NFATc2 expression was increased in both spleen and MLNs, and $\mathrm{CD}^{+} \mathrm{T}$ cells derived from these tissues. NFATc2 may be involved in the mechanism of food allergy. 


\title{
0-83 Human CD4+ T cell-mediated cutaneous-GVHD in hIL-1 $\gamma / \mathrm{IL}-23$ transgenic NOG mice.
}

\author{
Ryoji Ito, Ikumi Katano, Takeshi Takahashi, Mika Yagoto, Kenji Kawai, Motohito \\ Goto, Tomoyuki Ogura, Riichi Takahashi, Mamoru Ito \\ Central Institute for Experimental Animals
}

Xenograft animal models using immunodeficient mice are worthwhile therapeutic animal models for drugs discovery. Recently, we developed cutaneous xeno-GVHD model using NOG mice transferred with human CD4 $\mathrm{T}$ cells, and they developed several symptoms of skin inflammation including alopecia, epidermal hyperplasia, and neutrophilia. Then, human CD4 T cells accumulated in the skin and differentiated into pathogenic Th17 cells which produce both IL-17 and IFN $\gamma$ cytokines. These cells were required for the skin symptoms through human IL-17dependent mechanisms in this model. In this study, we generated a novel NOG mouse strain bearing human IL-1 $\beta$ and IL-23 cytokines that are critical inducers for Th17 cells to accelerate the cutaneous xeno-GVHD. Transplantation of human CD4 $\mathrm{T}$ cells into these NOG IL- $1 \beta / 23$ $\mathrm{Tg}$ mice showed markedly increase the alopecia symptoms and immediately died compared to control NOG mice. Highly infiltrated human CD4 $\mathrm{T}$ cells were observed in the skin but not in the spleen of Tg mice. Furthermore, human CD4 T cells were significantly differentiated into the effector memory phenotype (CD62L-CD45RA-) in $\mathrm{Tg}$ mice but not in nonTg mice, suggested human IL-1 $\beta$ and IL-23 activated CD4 T cells to enhance the cutaneous xeno-GVHD symptoms. Taken together, the NOG IL-1 $\beta$ /23 Tg mice may be useful for the preclinical tool in development of therapeutic drugs targeting human Th17 cells for prevention of cutaneous-GVHD.

\section{0-84 Analysis of human leucocytes in pregnant humanized mice}

\author{
$\bigcirc$ Yusuke Ohno ${ }^{1}$, Mika Kojima ${ }^{1}$, Rihito Kinami1, Mamoru Ito $^{2}$, Yoshie Kametani ${ }^{1}$ \\ ${ }^{1}$ Department of Molecular Life Science, Tokai University School of Medicine, Isehara, Japan ${ }^{2}$ Central \\ Institute for Experimental Animals, Kawasaki, Japan
}

Female constructs a unique immune system during pregnancy to accept allogeneic fetus. Pregnancy associated hormone, progesterone, plays a role in the immune system. Because there are no experimental models to investigate human specific pregnant immunity in vivo, we tried to establish a pregnant humanized mouse system as a model of human pregnant immunity. Human PBMCs were transplanted into female NOG mice immediately after plug recognition. After 14 days, the cell suspensions were prepared from the mouse lymphoid tissues and placenta to conduct Flow cytometry (FACS). Plasma proteins were analyzed by LC/MS and Plasma progesterone (P4) level was measured by ELISA. The concentration of P4 in the pregnant mouse plasma was in average $3.02 \mathrm{ng} / \mathrm{ml}$ at Day 14, which was 18.2 times higher than nonpregnant mice. The level was equivalent to wild type mouse, but lower than that of human 3rd trimester (100-150 ng/ml). Cell number of total leucocytes tended to increase in the spleen, along with B cell numbers in the pregnant mouse. Although most of the B cells expressed the markers of plasmablast, an antibodysecreting subset, the level of IgG in the plasma was significantly lower than non-pregnant mice. Activation of human Th cells was suppressed dependent on the $\mathrm{P} 4$ concentration in vitro, suggesting that $\mathrm{P} 4$ participates in the suppression of antibody production of $\mathrm{B}$ cells in pregnant humanized mice. 


\title{
0-85 Establishment of a humanized mouse model for HTLV-1 infection and evaluation of antiviral drugs
}

\author{
Kazu Okuma, Kenta Tezuka, Madoka Kuramitsu, Sahoko Matsuoka, Isao Hamaguchi \\ Department of Safety Research on Blood and Biological Products, National Institute of Infectious \\ Diseases, Tokyo, Japan
}

Human T-cell leukemia virus type 1 (HTLV-1) causes refractory diseases including adult $\mathrm{T}$-cell leukemia in a part of infected individuals after a long latent infection. However, antiviral therapies and infectious animal models for evaluation of drugs are not sufficiently established. Thus, in this study we aimed to establish a humanized mouse model for HTLV-1 infection. We purified $\mathrm{CD}_{133^{+}}$hematopoietic stem cells from human cord blood, and transplanted these cells into the liver of newborn severe immunodeficient mice. After around 16 weeks human $\mathrm{T}$ cells efficiently grew in the peripheral blood, indicating humanization. Therefore, we inoculated these humanized mice with lethally irradiated, HTLV- 1-infected cell line, MT-2 ip. Subsequently, we found rapid proliferation of HTLV-1-infected $\mathrm{CD} 4^{+} \mathrm{CD} 25^{+}$cells as shown by flow cytometry and a marked increase in HTLV-1 proviral load (PVL) as demonstrated by quantitative PCR in the peripheral blood. In addition we administered our anti-HTLV-l drug candidate, a recombinant vesicular stomatitis virus (rVSV), to these mice ip. This candidate significantly inhibited proliferation of HTLV-1-infected cells and elevation of PVL, suggesting efficacy of rVSV. Taken together, our present findings indicate that HTLV-1-infected humanized mice represent an excellent animal model for HTLV-1 infection and provide a useful tool for evaluation of antiviral drugs.

\section{0-86 Bone marrow-derived mesenchymal stromal cells protect lung allograft from acute rejection.}

\author{
Masahiko Kanehira ${ }^{1}$, Naoya Ishibashi², Tatsuaki Watanabe ${ }^{2}$, Masafumi Noda ${ }^{1,2}$, \\ Yoshinori Okada ${ }^{1,2}$ \\ ${ }^{1}$ Advanced Medical Training Center, Tohoku University Hospital, Miyagi, Japan ${ }^{2}$ Department of \\ Thoracic Surgery, Tohoku University Institute of Development Aging and Cancer, Miyagi, Japan
}

Lung transplantation has been established as an effective therapeutic modality for patients with end-stage lung diseases. In spite of rapid advances in surgical techniques and perioperative care, the 5-year survival rate after lung transplantation remains at approximately $50 \%$. An important cause of morbidity and mortality in lung transplantation patients is allograft rejection. In this study, we explored the potential of hMSCs to inhibit acute rejection of lung allografts by using a rat model of lung allograft transplantation. Our results demonstrated that administration with hMSCs once (day 0) or twice (days 0 and 3) (hereinafter hMSCl and hMSC2, respectively) into post-surgical rats significantly protected lung grafts from acute rejection compared to untreated controls. It is noteworthy that rats administered hMSC 2 after surgery exhibited the mildest rejection of all treatments. Interestingly, the mRNA level of IL-17A was significantly down-regulated, and conversely, the mRNA levels of PD-Ll was significantly upregulated in allografts by hMSC2 administration. Future studies using hMSCs will be undertaken to establish the long-term acceptance of lung allografts with no rejection. 

via downregulation of PPARalpha

\author{
$\bigcirc$ Haruo Hashimoto ${ }^{1}$, Masafumi Yamamoto ${ }^{1}$, Emika Sugiura ${ }^{1}$, Hayato Abe ${ }^{1}$, \\ Takahiro Kagawa ${ }^{1}$, Motohito Goto ${ }^{1}$, Ri-ichi Takahashi ${ }^{1}$, Toshio Akimoto², Hiroshi Suemizu ${ }^{1}$ \\ ${ }^{1}$ Laboratory Animal Department, Central Institute for Laboratory Animals ${ }^{2}$ Division of Laboratory \\ Animal Science, Nippon Medical School
}

Expression of peroxisome proliferator-activated receptor (PPAR) alpha was investigated in adiponectin knockout mice to elucidate the relationship between PPAR alpha and adiponectin deficiency-induced diabetes. Adiponectin knockout $\left(\mathrm{Adp}^{-/}\right)$mice were generated by gene targeting. Glucose tolerance test (GTT), insulin tolerance test (ITT), and organ sampling were performed in $\mathrm{Adp}^{-/}$mice at the age of 10 weeks. PPAR alpha, insulin, triglyceride, free fatty acid (FFA), and tumor necrosis factor alpha
(TNFalpha) were analyzed from the sampled organs. Adp ${ }^{-/-}$mice showed impaired glucose tolerance and insulin resistance. Additionally, PPAR alpha levels were decreased and plasma concentration of triglyceride, FFA, and TNF alpha were increased. These data indicate that insulin resistance in $\mathrm{Adp}^{-/}$mice is likely caused by an increase in TNFalpha concentration via downregulation of PPAR alpha.

\title{
0-88
}

Effects of environmental enrichment on pathogenesis and autonomic nervous functions in diabetic mice

\author{
$\bigcirc$ Kentaro Kaneko ${ }^{1,2}$, Akitoshi Chikamoto ${ }^{1}$, Shin-ichi Sekizawa ${ }^{1}$, Machiko Yamamoto², \\ Masayoshi Kuwahara ${ }^{1}$ \\ ${ }^{1}$ Veterinary Pathophysiology and Animal Health, Graduate school of Agricultural and Life Sciences, \\ The University of Tokyo ${ }^{2}$ School of Nursing, Ibaraki Christian University, Ibaraki, Japan
}

Environmental enrichment (EE) affects improving anxiety and stress in experimental animals, while little is known about the influence on autonomic nervous function which might be closely related to the pathogenesis of diabetes mellitus. The aim of this study was to clarify the influence of $\mathrm{EE}$ on the pathogenesis and autonomic nervous functions in the NSY mouse, type 2 diabetes model. All mice were kept individually and supplied water and food ad libitum. The control group was kept under normal bedding conditions, while nesting materials were added in the EE group. Electrocardiograms in both groups were recorded using telemetry system for
4 weeks after setting the nesting material. The autonomic nervous function was evaluated by power spectral analysis of heart rate variability and the HF region is known as a representative of parasympathetic nervous activity. Both groups showed lower heart rate and higher HF power in the light period as compared with the dark period, while HF power in the EE group, especially in the light period, tended to increase during recording periods and became significantly higher than that in the control group in the end. These results suggest that environmental enrichment may suppress the pathogenesis of diabetes by increasing parasympathetic activity. 


\title{
0-89 \\ Phenotypic analysis of a novel murine model of Congenital diabetes: the insulin hyposecretion mouse
}

\author{
$\bigcirc$ Kenta Nakano ${ }^{1,2}$, rieko Takanashi ${ }^{1}$, Hayato Sasaki ${ }^{2}$, Yuki Takahashi ${ }^{2}$, Yukiko Shimizu ${ }^{1}$, \\ Tadashi Okamura ${ }^{1}$, Nobuya Sasaki ${ }^{2}$ \\ ${ }^{1}$ Research Institute National Center for Global Health and Medicine, Tokyo, Japan ${ }^{2}$ Kitasato Graduate \\ School of Veterinary Medicine, Aomori, Japan
}

Diabetic animal models have made an enormous contribution to our understanding of the etiology of diabetes and the development of new medications for diabetes. We have established a novel, nonobese murine strain with spontaneous diabetes, the insulin hyposecretion (ihs) mouse. The incidence of diabetes in the males was about 30\% at 12 weeks of age and $90 \%$ at 42 weeks, while none of the females developed diabetes. The ihs mice had significantly higher blood glucose levels than those of control mice throughout the oral glucose tolerance test, whereas the insulin tolerance test showed that ihs mice had normal insulin sensitivity. The islets from mutants showed a normal shape with a smooth periphery and the insulin content of the mutant pancreata was comparable to that of the control mice. Thus, the dysfunction in insulin secretion was not caused by defects in insulin production or islet architecture and organization. The pancreata perfusion experiments indicated that ihs mice showed a marked impairment of insulin secretion due to stimulation by high levels of glucose and potassium chloride. The unique characteristic of the ihs mouse is that it allows not only the analysis of the progression of diabetes, but also the identification of unknown genes involved in insulin secretion.

\section{$0-90$ \\ Plasma biomarker for development and progression of coronary atherosclerosis in WHHLMI rabbits.}

$\bigcirc$ Masashi Shiomi ${ }^{1,2}$, Hiroaki Takeda ${ }^{3}$, Yasuhiro Irino ${ }^{4}$, Ying $\mathrm{Yu}^{1}$, Tomonari Koike ${ }^{1}$, Yosihiro Izumi $^{3}$, Masakazu Shinohara ${ }^{5}$, Takeshi Bamba ${ }^{3}$, Tatsuro Ishida ${ }^{6}$

${ }^{1}$ Institute for Experimental Animals, Kobe University Graduate School of Medicine, Kobe, Japan ${ }^{2}$ Comparative Pathophysiolosy, Kobe University Graduate School of Medicine, Kobe, Japan ${ }^{3}$ Research Center for Transomics Medicine, Medical Institute of Bioregulation, Kyushu University, Fukuoka, Japan ${ }^{4}$ Evidence-based Laboratory Medicine, Kobe University Graduate School of Medicine, Kobe, Japan ${ }^{5}$ Epidemiology, Kobe University Graduate School of Medicine, Kobe, Japan ${ }^{6}$ Cardiovascular Medicine, Kobe University Graduate School of Medicine, Kobe, Japan

[Purpose] The purpose of this study is to identify biomarkers for coronary lesions.[Methods] Ultracentrifugation of plasma lipoproteins, metabolome analyses and lipidome analyses of plasma were performed. Coronary lesions were evaluated by cross-sectional narrowing. The severity of aortic lesions was evaluated by percentage of lesion area on aortic surface. [Results] Despite almost no differences in aortic lesions and lipoprotein lipids, the extent of coronary plaque showed large individual differences. In metabolome analyses, the relative concentration of $\mathrm{N}$-forminylglucose was high in rabbits with mild coronary lesions. In lipidome analyses, the relative concentrations of ceramide were high, but were low in triacylglycerol (TAG) 44:1 and TAG 48:8 in rabbits with severe coronary lesions. [Conclusion] Parameters detected in metabolome or lipidome analyses might be markers for coronary lesions. 


\title{
0-91 The Generation of Novel SCID Mice Mediated by CRISPR/Cas9
}

\author{
Suvd Byambaa ${ }^{1}$, Hideki Uosaki ${ }^{1}$, Hiromasa Hara ${ }^{1}$, Hiroaki Shibata ${ }^{1}$, Tomoyuki Abe ${ }^{1}$, \\ Yasumitsu Nagao ${ }^{2}$, Osamu Nureki ${ }^{3}$, Tsukasa Ohmori ${ }^{4}$, Yutaka Hanazono \\ ${ }^{1}$ Division of Regenerative Medicine, Jichi Medical University ${ }^{2}$ Center for Experimental Medicine, Jichi Medical University、 \\ ${ }^{3}$ Department of Biological Sciences, The University of Tokyo ${ }^{4}$ Department of Biochemistry, Jichi Medical University
}

The CRISPR/Cas9 is giving opportunities to develop animal models for human diseases with specific mutations regardless of species. X-SCID mice can be used as a model for targeted genome editing approach or as a base for humanized mice. In X-SCID patients, mutations have been identified in all 8 exons of the $I 12 \mathrm{rg}$ gene and the majority of mutations are point mutations. In contrast, commercially available NOG and NSG mice recapitulate SCID phenotype, however, their mutations are not suitable for a genome editing model. Therefore, we generated novel SCID mouse strains mimicking X-SCID patients with small insertion/deletion mutations in II2rg using CRISPR/Cas9. To generate SCID mice, SpCas 9 mRNA and gRNA targeting the exon 2,3 or 4 of $I 12 \mathrm{rg}$ were injected into fertilized mouse eggs. In the F0 generation, we obtained 35 pups and 25 out of them were positive for Surveyor assay, and by sequencing, $7 \mathrm{bp}$ deletion was detected in strain targeting the exon 2 and $1 \mathrm{bp}$ insertion in strains targeting the exons 3 and 4. We also confirmed the lack of $\mathrm{T}$ and B lymphocytes in the peripheral blood. These data indicated that they were genetically and phenotypically SCID. In summary, we generated novel strains of SCID mice with small mutations in $I I 2 \mathrm{rg}$ by CRISPR/Cas9.

\section{$0-92$ The effect of reactivation in latent infection model of herpes virus to cognitive function in SAMP8}

\author{
Seiichi TANAKA ${ }^{1}$, Yuko TAKAYASHIKI ${ }^{2}$, Hiroshi NAGASHIMA ${ }^{2}$ \\ ${ }^{1}$ Center for Experimental Animals, Fukuoka University, Fukuoka, Japan ${ }^{2}$ Animal-care Co., Ltd., Tokyo, \\ Japan
}

(Aim) We are researching in latent infection model of swine herpes virus (Pseudorabies virus) to confirm the report of relation between Alzheimer's disease and infection with herpes simplex virus. In this test, we used SAM P8 mice which showed decrease cognitive function more remarkable than SAM P1 mice and considered cognitive function after reactivation of latent infection virus.

(Methods)We administered a serum of pigs anti Pseudorabies virus to SAM P8 and challenged with 100 LD $_{50}$ of YS-81. We regarded mice existed over two months as latent infected mice. We considered virus reactivation test after two months or six months of the infection and detected virus DNA by PCR from nasal swabs.
After virus reactivation test of six months, we conducted Y's maze test and object recognition test. After the test, we harvested brain samples from all mice and detected $\beta$-amyloid by ELISA.

(Results and discussion) In Y's maze test and object recognition test, we admitted significantly restraint of decline in cognitive function only when we reactivated the virus in two months of the infection. In the group reactivated in six months of the infection, there was no difference significantly between only latentinfection group and no-infection group. From the above, Alzheimer's disease is improved in latently infected SAM P8 mice when the virus is reactivated in two months of infection. 


\title{
0-93 Novel Mutation in a Member of the Src Family Kinases Causes Autoinflammatory Bone Disease in Mice
}

\author{
Koichiro Abe ${ }^{1}$, Nobuhiko Takamatsu ${ }^{2}$ \\ ${ }^{1}$ Department of Molecular Life Science, Tokai University School of Medicine ${ }^{2}$ School of Science, \\ Kitasato University ${ }^{3}$ Kumamoto University
}

Autoinflammatory bone diseases are associated with subsequent episodes of acute bone pain caused by abnormal innate immune response. In a mouse ENU mutagenesis screen, the Alils mutant strain was isolated by spontaneous inflammation on peripheral paws, and showed systemic reduction in bone density. By combination of genetic mapping and candidate sequencing, we found a missense mutation in the C-terminal regulatory tail region of a member of Src family tyrosine kinases (SFKs). For functional confirmation, we have employed CRISPR/ Cas9-mediated genome editing of the SFK at the N-terminus to generate compound mutant
Alil8 mice. Because its knockout mice are morphologically normal, we can verify whether the Alil8 arthritic phenotype depends on the SFK or not. Consequently, N-terminal frame shift mutations and ATG or splice acceptor deletion in Ali18 mice abolish the arthritic phenotype (10/16 strains), but N-terminal inframe mutations exhibit swollen paws (6/16 strains). In addition, an in vitro kinase assay of the SFK by Csk, the C-terminal Src kinase, revealed that inhibitory phosphorylation is reduced compared to that of wild type. Our results strongly indicate that activated SFK-mediated signaling is involved in autoinflammatory bone diseases.

\section{0-94 Quantitative Evaluation of Drug Efficacy by Acoustic Rhinometry in Allergic Rhinitis Model Macaque}

\author{
$\bigcirc$ Fusako Mitsunaga $^{1,2}$, Shin Nakamura ${ }^{1,2}$, Motofumi Ohki ${ }^{3}$ \\ ${ }^{1}$ Biomedical Institute, NPO Primate Agora ${ }^{2}$ I Tech Lab. Inc. ${ }^{3}$ Department of Otorhinolaryngology, \\ Kitasato University Medical Center
}

Macaque monkeys are an invaluable non-human primate (NHP) model for allergic rhinitis because of their high similarity with humans in their immunological and anatomical nature. Macaques exhibit nasal congestion responding to allergen, moreover, they closely resemble humans in the morphology of their nasal cavities compared with rodents. Rhinoscopy is effective in direct observation of nasal congestion, however it is not quantitative. Acoustic rhinometry is a noninvasive technique to measure nasal congestion using acoustic reflections. We have reported the application of the acoustic rhinometry on an NHP model of allergic rhinitis for the first time [1]. In the present study, we aimed at advancing the applicability of the technique to evaluate drugs.
We established an allergic rhinitis model of cynomolgus macaque (Macaca fascicularis) by sensitization with Japanese cedar pollen. Increased levels of allergen-specific IgE were monitored by ELISA. Individuals exhibited nasal reaction against allergen were used as model monkeys.

We applied acoustic rhinometry to measure nasal congestion and quantified the extent of congestion as the minimal cross-sectional area (MCA). We then utilized this technique to evaluate the efficacy of commercially available drugs. Thus, the acoustic assessment achieved quantitative evaluation of the drug efficacy in NHP model.

[1] Mitsunaga F. et al., 2017, JBM, 5: 27-32. 